\title{
Charmonia and Bottomonia in a Magnetic Field
}

\author{
Jeremy Alford and Michael Strickland \\ Department of Physics, Kent State University, Kent, OH 44242 United States
}

(Dated: August 5, 2021)

\begin{abstract}
We study the effect of a static homogeneous external magnetic field on charmonium and bottomonium states. In an external magnetic field, quarkonium states do not have a conserved centerof-mass momentum. Instead there is a new conserved quantity called the pseudomomentum which takes into account the Lorentz force on the particles in the system. When written in terms of the pseudomomentum, the internal and center-of-mass motions do not decouple and, as a result, the properties of quarkonia depend on the states' center-of-mass momentum. We analyze the behavior of heavy particle-antiparticle pairs subject to an external magnetic field assuming a three dimensional harmonic potential and Cornell potential plus spin-spin interaction. In the case of the Cornell potential, we also take into account the mixing of the $\eta_{c}$ and $J / \psi$ states and $\eta_{b}$ and $\Upsilon$ states due to the background magnetic field. We then numerically calculate the dependence of the masses and mixing fractions on the magnitude of the background magnetic field and center-of-mass momentum of the state.
\end{abstract}

PACS numbers: 11.15Bt, 04.25.Nx, 11.10Wx, 12.38Mh

Keywords: Quarkonia, Non-relativistic QCD, Magnetic Field, Pseudomomentum 


\section{INTRODUCTION}

The behavior of matter subject to magnetic fields has been a subject of interest for physicists for quite some time. Already over one hundred years ago Pieter Zeeman showed that an external magnetic field affected the spectrum of light emitted by a flame [1-3]. In recent years there has been considerable attention focused on the question of what happens to matter in the presence of extremely strong magnetic fields. There are at least two situations in which extremely strong magnetic fields are expected to be generated: (1) During early times after non-central heavy ion collisions one expects $B \sim m_{\pi}^{2} \sim 10^{18}$ Gauss at energies probed by the Relativistic Heavy Ion Collider (RHIC) and $B \sim 15 m_{\pi}^{2} \sim 1.5 \times 10^{19}$ Gauss at Large Hadron Collider (LHC) energies [4 9] and (2) in the interior of magnetars, which are a class of neutron stars which possess magnetic fields on the order of $10^{18}-10^{19}$ Gauss [10]. In this paper, we study the behavior of charmonium and bottomonium states subject to magnetic fields with an eye towards applications to the phenomenology of relativistic heavy ion collisions.

Interest in the effects of strong magnetic fields in heavy ion collisions has become a hot topic recently following the prediction of a non-trivial quantum chromodynamics (QCD) effect dubbed "the chiral magnetic effect" which stems from small P- and CP-odd interactions inducing an electromagnetic current when a quark-gluon plasma (QGP) is placed in an external magnetic field [4]. There has been much work related to this in recent years and in addition it has been shown how to self-consistently take into account this effect through Berry curvature flux in the presence of a magnetic field [11, 12]. The existence of such high magnetic fields has also prompted many research groups to study how the finite temperature deconfinement and chiral phase transitions are affected by the presence of a strong background magnetic field. These studies have included direct numerical investigations using lattice QCD [13 17] and theoretical investigations using a variety of methods including, for example, perturbative QCD studies, model studies, and string-theory inspired anti-de Sitter/conformal field theory (AdS/CFT) correspondence studies [18 41].

In this paper, we consider the effects of magnetic fields on heavy quarkonium states, focussing on 1 s charmonium and bottomonium states. The physics of quantum mechanical bound states in a background magnetic field is complicated by the fact that in a background magnetic field the center-of-mass (COM) momentum is not a conserved quantity due to the 
breaking of translational invariance. Instead one must take into account the Lorentz force on the constituents and construct a quantity called the COM pseudomomentum [42 55]. However, in practice one finds that, even after expressing the Hamiltonian in terms of the pseudomomentum, it is not possible to factorize the Hamiltonian into free COM motion plus decoupled internal motion. As a result, the spectrum of a bound state in background magnetic field depends on the COM momentum of the system. To the best of our knowledge, the first theoretical consideration of motional effects was by Lamb [44] and as we will show this effect is related to the so-called motional Stark effect.

In this paper we investigate the effect of strong magnetic fields on heavy quarkonium states including such motional effects. Heavy quarkonium is a nice test bed for QCD since heavy quark states are dominated by short distance physics and can be treated using heavy quark effective theory [56]. Based on such effective theories of QCD, non-relativistic quarkonium states can be reliably described. Their binding energies are much smaller than the quark mass $m_{q} \gg \Lambda_{\mathrm{QCD}}(q=c, b)$, and their sizes are much larger than $1 / m_{q}$. Since the velocity of the quarks in the bound state is small $(v \ll c)$, quarkonium can be understood in terms of non-relativistic potential models such as the Cornell potential which can be derived directly from QCD using effective field theory [57 [59].

We present numerical calculations using a Cornell potential supplemented by a spin-spin interaction which allows for a splitting between the spin-singlet and spin-triplet states. This study contributes to ongoing discussions of the effect of strong magnetic fields on QCD bound states [60 66]. Apart from the long range interactions, which are fundamentally different, the physics of heavy quarkonium is very similar to positronium [67 79] In addition to motional effects [79], it is also necessary to take into account the hyperfine mixing in the background magnetic field. In positronium this results in a change of the spin-singlet and spin-triplet energy eigenvalues and "quenching" of ortho-positronium $3 \gamma$ decays [74, 75, 77]. Analogous effects occur in quarkonium and we present quantitative calculations of the effect including a realistic heavy quark interaction potential. In addition, we present exact analytic formulas which can be obtained assuming a harmonic interaction between the constituents. The harmonic interaction results are used for purposes of discussion and also to check the numerical methods which are applied in the more realistic case.

The structure of the paper is as follows. In Sec. II we introduce the pseudomomentum.

\footnotetext{
${ }^{1}$ For a nice review of positronium physics see Ref. [80].
} 
In Sec. III we discuss the application to two particle states and then specialize to the case of particle-antiparticle states. In Sec. IV we discuss the relation of the pseudopotential derived in the previous section to the motional Stark effect. In Sec. V we discuss the prescription we use to subtract the energy associated with the center of mass motion. In Sec. VI we discuss the mixing of the spin singlet and triplet states in the presence of a magnetic field. In Sec. VII we present the potential we use for our final results. In Sec. VIII we present our numerical results. In Sec. IX] we present our conclusions and outlook for the future. In three appendices we collect details of the inter-quark potential used and resulting spectra, our numerical method for solving the $3 \mathrm{~d}$ Schrödinger equation, and an investigation of what happens to a harmonic state with a given center of mass momenta when a magnetic field is turned on suddenly.

\section{PARTICLE IN A CONSTANT MAGNETIC FIELD}

We begin with the basics by introducing the pseudomomentum in the context of a single classical non-relativistic charged spin one-half particle in a background magnetic field. As we will demonstrate, unlike the particle momentum, the pseudomomentum is conserved since it takes into account the Lorentz force on the particle. The classical non-relativistic Hamiltonian for a particle in a constant magnetic field can be written

$$
\mathcal{H}=\frac{1}{2 m}[\mathbf{p}-q \mathbf{A}(\mathbf{r})]^{2}+V(\mathbf{r})-\boldsymbol{\mu} \cdot \mathbf{B}+m,
$$

where $m$ is the rest mass of the particle and we assume $\mathbf{B}(\mathbf{x})=(0,0, B)$ which, in symmetric gauge, can be expressed in terms of the vector potential $\mathbf{A}(\mathbf{r})=\frac{1}{2} \mathbf{B} \times \mathbf{r}=\frac{1}{2} B(-y, x, 0)$.

We can apply Hamilton's equations to derive the equation of motion

$$
\begin{aligned}
-\frac{\partial \mathcal{H}}{\partial r_{i}} & =\dot{p}_{i}, \\
\frac{\partial \mathcal{H}}{\partial p_{i}} & =\dot{r}_{i} .
\end{aligned}
$$

The second Hamilton equation gives $m \dot{r}_{i}=p_{i}-q A_{i}$ which allows us to solve for the canonical momentum, $p_{i}=m \dot{r}_{i}+q A_{i}$. Using this, we can evaluate the full time derivative of the canonical momentum

$$
\begin{aligned}
\dot{p}_{i} & =m \ddot{r}_{i}+q\left(\frac{\partial A_{i}}{\partial t}+\frac{d r_{j}}{d t} \frac{\partial A_{i}}{\partial r_{j}}\right), \\
& =m \ddot{r}_{i}+q \dot{r}_{j} \frac{\partial A_{i}}{\partial r_{j}}
\end{aligned}
$$


where, in going from the first to second line we have used the fact that the vector potential is static in the case under consideration. The right hand side of the first Hamilton equation gives

$$
\begin{aligned}
-\frac{\partial \mathcal{H}}{\partial r_{i}} & =\frac{1}{m}(\mathbf{p}-q \mathbf{A}(\mathbf{r})) \cdot\left(q \frac{\partial \mathbf{A}}{\partial r_{i}}\right)-\frac{\partial V}{\partial r_{i}}, \\
& =q \dot{r}_{j} \frac{\partial A_{j}}{\partial r_{i}}-\frac{\partial V}{\partial r_{i}} .
\end{aligned}
$$

Equating the two sides we obtain

$$
m \ddot{r}_{i}=q \dot{r}_{j} \frac{\partial A_{j}}{\partial r_{i}}-q \dot{r}_{j} \frac{\partial A_{i}}{\partial r_{j}}-\frac{\partial V}{\partial r_{i}}
$$

Using $\mathbf{v} \times \mathbf{B}=\dot{\mathbf{r}} \times(\nabla \times \mathbf{A})=\nabla(\dot{\mathbf{r}} \cdot \mathbf{A})-(\dot{\mathbf{r}} \cdot \nabla) \mathbf{A}$ we can rewrite this as

$$
m \ddot{\mathbf{r}}=q \dot{\mathbf{r}} \times \mathbf{B}-\nabla V .
$$

In the case that the there is no potential, $V=0$, we have only the Lorentz force acting on the particle

$$
m \ddot{\mathbf{r}}=q \dot{\mathbf{r}} \times \mathbf{B},
$$

which shows that the momentum is not conserved in a constant magnetic field, as expected; however, we can introduce a quantity which is conserved called the pseudomomentum, $\mathcal{K}$,

$$
\begin{aligned}
\mathcal{K} & =m \dot{\mathbf{r}}+q \mathbf{B} \times \mathbf{r}, \\
& =\mathbf{p}+\frac{q}{2} \mathbf{B} \times \mathbf{r}, \\
& =\mathbf{p}+q \mathbf{A},
\end{aligned}
$$

such that the equation of motion can be expressed as

$$
\frac{d}{d t} \mathcal{K}=0 \text {. }
$$

\section{TWO COUPLED PARTICLES IN A CONSTANT MAGNETIC FIELD}

We next consider the case of two particles subject to a translationally invariant potential in non-relativistic quantum mechanics. We will follow closely the treatment found in Ref. [53]. The Hamiltonian operator for two particles in a constant magnetic field can be written as

$$
\mathcal{H}=\frac{1}{2 m_{1}}\left[\mathbf{p}_{1}-q_{1} \mathbf{A}\left(\mathbf{r}_{1}\right)\right]^{2}+\frac{1}{2 m_{2}}\left[\mathbf{p}_{2}-q_{2} \mathbf{A}\left(\mathbf{r}_{2}\right)\right]^{2}+V\left(\mathbf{r}_{1}-\mathbf{r}_{2}\right)-\boldsymbol{\mu} \cdot \mathbf{B}+m_{1}+m_{2},
$$


where $\boldsymbol{\mu}=\boldsymbol{\mu}_{1}+\boldsymbol{\mu}_{2}$ is the sum of the two particles' magnetic moments and $\mathbf{B}(\mathbf{x})=(0,0, B)$, which can be expressed in terms of the vector potential $\mathbf{A}(\mathbf{r})=\frac{1}{2} \mathbf{B} \times \mathbf{r}=\frac{1}{2} B(-y, x, 0)$ in symmetric gauge. As usual, $\mathbf{p}_{i}=-i \nabla$ is the momentum operator for the $i^{\text {th }}$ particle. As in the previous section, one finds that the COM momentum of the system is no longer conserved. This is due to the breaking of translational invariance by the vector potential (changing the origin changes $\mathbf{A}$ ). In order to preserve translational invariance in a constant magnetic field an additional gauge transformation is required. This can be achieved by introducing the generalized pseudomomentum operator [53]

$$
\mathcal{K}_{k}=\sum_{j=1}^{2}\left(-i \frac{\partial}{\partial x_{j k}}-q_{j} \int_{0}^{\mathbf{r}_{j}} \frac{\partial \mathbf{A}}{\partial x_{k}} \cdot d \mathbf{r}\right)
$$

where $k=1,2,3$ denotes cartesian components. Integrating and discarding a constant one obtains

$$
\mathcal{K}=\sum_{j=1}^{2}\left(\mathbf{p}_{j}-q_{j} \mathbf{A}_{j}+q_{j} \mathbf{B} \times \mathbf{r}_{j}\right)
$$

In the gauge used herein we have $\mathbf{A}(\mathbf{r})=\frac{1}{2} \mathbf{B} \times \mathbf{r}$ which allows us to simplify this to

$$
\begin{aligned}
\mathcal{K} & =\sum_{j=1}^{2}\left(\mathbf{p}_{j}+\frac{1}{2} q_{j} \mathbf{B} \times \mathbf{r}_{j}\right) \\
& =\sum_{j=1}^{2}\left(\mathbf{p}_{j}+q_{j} \mathbf{A}_{j}\right)
\end{aligned}
$$

which is the generalization of the one particle case obtained in the previous section. One can verify explicitly that the pseudomomentum operator commutes with the Hamiltonian

$$
[\mathcal{K}, \mathcal{H}]=0
$$

One can also compute the commutator of two components of $\mathcal{K}$ in which case one obtains

$$
\left[\mathcal{K}_{k}, \mathcal{K}_{l}\right]=-i \varepsilon_{k l m} B_{m}\left(\sum_{j=1}^{2} q_{j}\right)
$$

which means that one will only be able to determine all components of $\mathcal{K}$ simultaneously for a electric charge neutral system. 


\section{A. Two particles with equal and opposite charge}

In this section we specialize to the case that $q_{1}=-q_{2}=q$. To proceed we introduce center of mass and relative coordinates

$$
\begin{aligned}
\mathbf{R} & =\frac{m_{1} \mathbf{r}_{1}+m_{2} \mathbf{r}_{2}}{M}, \\
\mathbf{r} & =\mathbf{r}_{1}-\mathbf{r}_{2},
\end{aligned}
$$

where $M=m_{1}+m_{2}$. As is standard, we can express the individual positions as

$$
\begin{aligned}
& \mathbf{r}_{1}=\mathbf{R}+\frac{\mu}{m_{1}} \mathbf{r}, \\
& \mathbf{r}_{2}=\mathbf{R}-\frac{\mu}{m_{2}} \mathbf{r},
\end{aligned}
$$

where $\mu=m_{1} m_{2} / M$ is the reduced mass.

This allows us to simplify the pseudomomentum operator

$$
\begin{aligned}
\mathcal{K} & =\sum_{j=1}^{2}\left(\mathbf{p}_{j}+\frac{1}{2} q_{j} \mathbf{B} \times \mathbf{r}_{j}\right) \\
& =-i\left(\frac{\partial}{\partial \mathbf{r}_{1}}+\frac{\partial}{\partial \mathbf{r}_{2}}\right)+\frac{1}{2} q \mathbf{B} \times\left(\mathbf{r}_{1}-\mathbf{r}_{2}\right), \\
& =-i \frac{\partial}{\partial \mathbf{R}}+\frac{1}{2} q \mathbf{B} \times \mathbf{r}
\end{aligned}
$$

Since the system is neutral, the full two-particle eigenfunctions $\Phi$ of the Hamiltonian are simultaneous eigenfunctions of all components $\mathcal{K}_{i}$ of the pseudomomentum with eigenvalues $K_{i}$. This allows us to factorize the full wavefunction

$$
\Phi(\mathbf{R}, \mathbf{r})=\exp \left[i\left(\mathbf{K}-\frac{1}{2} q \mathbf{B} \times \mathbf{r}\right) \cdot \mathbf{R}\right] \Psi(\mathbf{r}) \equiv \phi(\mathbf{R}, \mathbf{r}) \Psi(\mathbf{r})
$$

which satisfies $\mathcal{K}_{j} \Phi=K_{j} \Phi$ by construction.

Expanding out the two-particle Hamiltonian one finds the "relative" Hamiltonian

$$
\begin{aligned}
\mathcal{H}_{\mathrm{rel}}=\frac{\mathbf{K}^{2}}{2 M}- & \frac{q}{M}(\mathbf{K} \times \mathbf{B}) \cdot \mathbf{r}+\frac{\mathbf{p}^{2}}{2 \mu}+\frac{q}{2}\left(\frac{1}{m_{1}}-\frac{1}{m_{2}}\right) \mathbf{B} \cdot(\mathbf{r} \times \mathbf{p}) \\
& +\frac{q^{2}}{8 \mu}(\mathbf{B} \times \mathbf{r})^{2}+V(\mathbf{r})-\boldsymbol{\mu} \cdot \mathbf{B}+m_{1}+m_{2},
\end{aligned}
$$

where $\mathbf{p}=-i \nabla$ is the relative momentum operator and one has the new eigenvalue equation $\mathcal{H}_{\text {rel }} \Psi(\mathbf{r})=E \Psi(\mathbf{r})$. Note that, unlike the case without the external field, the energy eigenvalue $E$ depends on the value of $\mathbf{K}$ through coupling in the second term and not only through the term $\mathbf{K}^{2} / 2 M$. 


\section{B. Heavy-light system}

In the limit that $m_{2} \rightarrow \infty$ while holding $m_{1}$ fixed, we have $M \rightarrow \infty$ and $\mu=m_{1} \equiv m$ and we obtain

$$
\mathcal{H}_{\mathrm{rel}}=\frac{\mathbf{p}^{2}}{2 m}-\frac{q}{2 m} \mathbf{B} \cdot(\mathbf{r} \times \mathbf{p})+\frac{q^{2}}{8 m}(\mathbf{B} \times \mathbf{r})^{2}+V(\mathbf{r})-\boldsymbol{\mu} \cdot \mathbf{B}+m,
$$

where we have discarded the infinite constant $m_{2}$ in this case. Recalling that $\mathbf{A}=\frac{1}{2} \mathbf{B} \times \mathbf{r}=$ $\frac{1}{2} B(-y, x, 0)$ one has $(\mathbf{B} \times \mathbf{r})^{2}=B^{2} \rho^{2}$ and using $\mathbf{B} \cdot(\mathbf{r} \times \mathbf{p})=(\mathbf{B} \times \mathbf{r}) \cdot \mathbf{p}=\rho B p_{\phi}=-i B \partial_{\phi}$ we obtain

$$
\mathcal{H}_{\mathrm{rel}}=-\frac{1}{2 m} \nabla^{2}+\frac{i}{2} \omega_{c} \frac{\partial}{\partial \phi}+\frac{m \omega_{c}^{2}}{8} \rho^{2}+V(\mathbf{r})-\boldsymbol{\mu} \cdot \mathbf{B}+m
$$

where $\omega_{c}=q B / m$. This is the standard non-relativistic Hamiltonian for a spin-one-half particle subject to a potential $V$ and an external magnetic field.

\section{Particle-antiparticle pair}

For a bound state consisting of a particle-antiparticle pair we have $m_{1}=m_{2}=m$, $M=2 m$, and $\mu=m / 2$. In this case the relative Hamiltonian simplifies to

$$
\mathcal{H}_{\mathrm{rel}}=\frac{\mathbf{K}^{2}}{2 M}-\frac{q}{M}(\mathbf{K} \times \mathbf{B}) \cdot \mathbf{r}-\frac{\nabla^{2}}{2 \mu}+\frac{q^{2}}{8 \mu}(\mathbf{B} \times \mathbf{r})^{2}+V(\mathbf{r})-\boldsymbol{\mu} \cdot \mathbf{B}+M .
$$

Next we decompose $\mathbf{K}=K_{x} \hat{\mathbf{x}}+K_{y} \hat{\mathbf{y}}+K_{z} \hat{\mathbf{z}}$ and simplify the expression above to obtain

$$
\mathcal{H}_{\mathrm{rel}}=\frac{\mathbf{K}^{2}}{2 M}+\frac{q B}{4 \mu} K_{x} y-\frac{q B}{4 \mu} K_{y} x-\frac{\nabla^{2}}{2 \mu}+\frac{q^{2} B^{2}}{8 \mu} \rho^{2}+V(\mathbf{r})-\boldsymbol{\mu} \cdot \mathbf{B}+M .
$$

\section{Relation between the pseudomomentum and kinetic center-of-mass momentum}

We now derive a general relation between the pseudomomentum and kinetic COM momentum. The COM kinetic momentum of the system is given by

$$
\begin{aligned}
\mathbf{P}_{\text {kinetic }} & =\sum_{j}\left(-i \frac{\partial}{\partial \mathbf{r}_{j}}-q_{j} \mathbf{A}_{j}\right), \\
& =-i \frac{\partial}{\partial \mathbf{R}}-\frac{1}{2} q \mathbf{B} \times \mathbf{r} .
\end{aligned}
$$

Therefore, we have

$$
\left\langle\mathbf{P}_{\text {kinetic }}\right\rangle=\frac{\int_{\mathbf{R}} \int_{\mathbf{r}} \Phi^{*}\left[-i \frac{\partial}{\partial \mathbf{R}}-\frac{1}{2} q \mathbf{B} \times \mathbf{r}\right] \Phi}{\int_{\mathbf{R}} \int_{\mathbf{r}} \Phi^{*} \Phi} .
$$


Using

$$
-i \frac{\partial}{\partial \mathbf{R}} \Phi=\left(\mathbf{K}-\frac{1}{2} q \mathbf{B} \times \mathbf{r}\right) \Phi
$$

one finds

$$
\left\langle\mathbf{P}_{\text {kinetic }}\right\rangle=\mathbf{K}-q \mathbf{B} \times\langle\mathbf{r}\rangle .
$$

\section{Particle-antiparticle pair with a harmonic interaction}

We now specialize to the case that the potential is harmonic in which case the wave functions and energy levels can be obtained analytically. Some of the results contained in this subsection were first obtained explicitly by Herold et al [53. We repeat the derivation here in order to use them as a basis for discussion of the COM momentum dependence of the energy. We also use this case as a check for our numerics since it can be solved analytically.

Using the general relative Hamiltonian for a particle-antiparticle pair (24) and $V(\mathbf{x})=$ $\frac{1}{2} k \mathbf{x}^{2}=\frac{1}{2} \mu \omega_{0}^{2}\left(x^{2}+y^{2}+z^{2}\right)$ we have

$$
\begin{aligned}
\mathcal{H}_{\mathrm{rel}} & =\frac{\mathbf{K}^{2}}{2 M}-\frac{\nabla^{2}}{2 \mu}+\frac{1}{2} \mu\left(\omega_{0}^{2}+\frac{\omega_{c}^{2}}{4}\right)\left(x^{2}+y^{2}\right)-\frac{\omega_{c} K_{y}}{4} x+\frac{\omega_{c} K_{x}}{4} y+\frac{1}{2} \mu \omega_{0}^{2} z^{2}-\boldsymbol{\mu} \cdot \mathbf{B}+M, \\
& =\frac{\mathbf{K}^{2}}{2 M}-\frac{\nabla^{2}}{2 \mu}+\frac{1}{2} a\left(x^{2}+y^{2}\right)-b x+c y+\frac{1}{2} d z^{2}-\boldsymbol{\mu} \cdot \mathbf{B}+M
\end{aligned}
$$

where $\omega_{c}=q B / \mu, \boldsymbol{\mu}=\boldsymbol{\mu}_{1}+\boldsymbol{\mu}_{2}, a=\mu\left(\omega_{0}^{2}+\omega_{c}^{2} / 4\right), b=\omega_{c} K_{y} / 4, c=\omega_{c} K_{x} / 4$, and $d=\mu \omega_{0}^{2}$.

We can rewrite the third, fourth, and fifth terms using

$$
\frac{1}{2} a\left(x^{2}+y^{2}\right)-b x+c y=\frac{1}{2} a\left[\left(x-\frac{b}{a}\right)^{2}+\left(y+\frac{c}{a}\right)^{2}\right]-\frac{1}{2 a}\left(b^{2}+c^{2}\right) .
$$

We can simplify things further by making use of a constant coordinate shift $\bar{x} \equiv x-b / a$ and $\bar{y} \equiv y+c / a$.

$$
\mathcal{H}_{\mathrm{rel}}=\frac{\mathbf{K}^{2}}{2 M}-\frac{\nabla^{2}}{2 \mu}+\frac{1}{2} a\left(\bar{x}^{2}+\bar{y}^{2}\right)+\frac{1}{2} d z^{2}-\frac{1}{2 a}\left(b^{2}+c^{2}\right)-\boldsymbol{\mu} \cdot \mathbf{B}+M
$$

which suggests that we use cylindrical coordinates with $\bar{x}=\rho \cos \phi, \bar{y}=\rho \sin \phi$, and $z=z$. After this, the eigenvalue equation $\mathcal{H}_{\text {rel }} \Psi=E \Psi$ becomes

$$
\left(-\frac{\nabla^{2}}{2 \mu}+\frac{1}{2} a \rho^{2}+\frac{1}{2} c z^{2}\right) \Psi(\mathbf{r})=\left(E-\frac{\mathbf{K}^{2}}{2 M}+\frac{b^{2}}{2 a}+\boldsymbol{\mu} \cdot \mathbf{B}+M\right) \Psi(\mathbf{r}) .
$$

Factorizing the relative wavefuction as $\Psi(\mathbf{r})=e^{i \ell \phi} Z(z) \psi(\rho)$ we find

$$
\left(-\frac{\partial^{2}}{\partial \rho^{2}}-\frac{1}{\rho} \frac{\partial}{\partial \rho}+\frac{|\ell|^{2}}{\rho^{2}}+\alpha^{4} \rho^{2}\right) \psi=2 \mu \lambda \psi
$$


where $\alpha^{2}=\sqrt{\mu a}=\mu \sqrt{\omega_{0}^{2}+\omega_{c}^{2} / 4}, \lambda=E-E_{z}-\mathbf{K}^{2} / 2 M+\left(b^{2}+c^{2}\right) / 2 a+\boldsymbol{\mu} \cdot \mathbf{B}+M$ and $E_{z}$ is the eigenvalue of the separated z-equation

$$
\left(-\frac{\partial^{2}}{\partial z^{2}}+\gamma^{4} z^{2}\right) Z=2 \mu E_{z} Z
$$

where $\gamma=(\mu c)^{1 / 4}=\sqrt{\mu \omega_{0}}$ which has a solution

$$
Z=N e^{-\frac{1}{2} \gamma^{2} z^{2}} H_{n_{z}}(\gamma z)
$$

and energy eigenvalue

$$
E_{z}=\left(n_{z}+\frac{1}{2}\right) \omega_{0}
$$

Convergence as $\rho \rightarrow \infty$ requires

$$
\lambda=\frac{\alpha^{2}}{\mu}\left(2 n_{\perp}+1+|\ell|\right)=\left(2 n_{\perp}+1+|\ell|\right) \sqrt{\omega_{0}^{2}+\frac{\omega_{c}^{2}}{4}} .
$$

Solving for $E$ we obtain the energy eigenvalues for the system

$$
E_{\mathbf{K}, n_{\perp} n_{z} \ell}=\frac{\mathbf{K}^{2}}{2 M}-\frac{\omega_{c}^{2}\left(K_{x}^{2}+K_{y}^{2}\right)}{32 \mu\left(\omega_{0}^{2}+\omega_{c}^{2} / 4\right)}+\left(n_{z}+\frac{1}{2}\right) \omega_{0}+\left(2 n_{\perp}+1+|\ell|\right) \sqrt{\omega_{0}^{2}+\frac{\omega_{c}^{2}}{4}}-\boldsymbol{\mu} \cdot \mathbf{B}+M .
$$

We can now write the full two-particle wave function

$$
\Phi_{\mathbf{K}, n_{\perp} n_{z} \ell}(\mathbf{R}, \mathbf{r})=\mathcal{N} \rho^{|\ell|} e^{i \ell \phi} e^{-\frac{1}{2} \gamma^{2} z^{2}} e^{-\frac{1}{2} \alpha^{2} \rho^{2}} H_{n_{z}}(\gamma z) L_{n_{\perp}}^{|\ell|}\left(\alpha^{2} \rho^{2}\right) e^{i\left(\mathbf{K}-\frac{1}{2} q \mathbf{B} \times \mathbf{r}\right) \cdot \mathbf{R}},
$$

where $\mathcal{N}$ is a normalization constant and

$$
\begin{aligned}
\omega_{c} & =\frac{q B}{\mu}, \\
\alpha^{2} & =\mu \sqrt{\omega_{0}^{2}+\frac{\omega_{c}^{2}}{4}}, \\
\beta & =\frac{\omega_{c}}{4 \mu\left(\omega_{0}^{2}+\omega_{c}^{2} / 4\right)}, \\
\gamma^{2} & =\mu \omega_{0}, \\
\rho^{2} & =\left(x-\beta K_{y}\right)^{2}+\left(y+\beta K_{x}\right)^{2}, \\
\phi & =\arctan \left(\frac{y+\beta K_{x}}{x-\beta K_{y}}\right) .
\end{aligned}
$$

Center-of-mass Kinetic Momentum

Using this we can analytically compute the relationship between the pseudomomentum and the COM kinetic momentum of the state. Using Eq. 28 and

$$
\mathbf{B} \times \mathbf{r}=B(-y, x, 0)=B\left(-\rho \sin \phi+\frac{c}{a}, \rho \cos \phi+\frac{b}{a}, 0\right),
$$


one finds in this case

$$
\left\langle\mathbf{P}_{\text {kinetic }}\right\rangle=\mathbf{K}-\frac{q B c}{a} \hat{\mathbf{x}}-\frac{q B b}{a} \hat{\mathbf{y}} .
$$

Plugging in the definitions of $a, b$, and $c$ we obtain

$$
\left\langle\mathbf{P}_{\text {kinetic }}\right\rangle=\left(\frac{4 \omega_{0}^{2}}{4 \omega_{0}^{2}+\omega_{c}^{2}} K_{x}, \frac{4 \omega_{0}^{2}}{4 \omega_{0}^{2}+\omega_{c}^{2}} K_{y}, K_{z}\right),
$$

As we can explicitly see from this expression, the components of the kinetic COM momentum do not directly correspond to the pseudomomentum components. We note that in App. C we derive this formula in a different manner by assuming a time-dependent magnetic field which turns on rapidly.

\section{RELATION TO THE MOTIONAL STARK EFFECT}

One way to intuitively understand the result obtained in Eq. (24) is try to derive it in a different manner. We can instead try to write down the non-relativistic Hamiltonian in the COM rest frame. This step is self-contradictory since, as we have pointed out previously, the COM momentum is not a conserved quantity in the presence of an external magnetic field; however, let's ignore this for the time being and assume that we can, in fact, boost to the rest frame of the state. As before, we assume that the magnetic field points in the $z$-direction and as a result the dynamics in the $z$ direction is straightforward. Putting the system at rest in the $z$-direction and assuming that we can also hold it at rest in the $y$-direction we need only consider boosts in the $x$ direction with $v_{x}=P_{x} / M$. In the lab frame there is only a magnetic field. In the co-moving frame there will be both electric and magnetic fields. Using the standard transformation laws for electric and magnetic fields one finds

$$
\begin{array}{ll}
E_{x}^{\prime}=0, & B_{x}^{\prime}=0, \\
E_{y}^{\prime}=-\gamma v_{x} B \approx-v_{x} B, & B_{y}^{\prime}=0, \\
E_{z}^{\prime}=0, & B_{z}^{\prime}=\gamma B \approx B,
\end{array}
$$

where for the terms with $\approx$ appearing we have discarded terms of the order $v_{x}^{2}$ and higher.

As we can see from the relations above, if we boost to the rest frame of the state, there is an additional electric interaction of the form $H_{\text {electric }}^{\prime}=-q \mathbf{E}^{\prime} \cdot \mathbf{r}$ where $\mathbf{r}=\mathbf{r}_{1}-\mathbf{r}_{2}$ is the relative position. Using the expressions above one finds trivially

$$
H_{\text {electric }}^{\prime}=\frac{q B}{4 \mu} P_{x} y
$$


where we have used the fact that for a particle-antiparticle system $M=4 \mu$. As we can see this is precisely the "extra term" in Eq. 24) (assuming $P_{x}=K_{x}$ and $P_{y}=K_{y}=0$ ). If we had allowed for a general direction for the COM momentum, we would have generated both terms. So we can see that the physical origin of these terms is, in fact, the motional Stark effect; however, deriving things in this manner we have blurred the important distinction between $\mathbf{P}$ and $\mathbf{K}$, where only the latter is a conserved quantity. In what follows we will simply use Eq. (24) since it is the correct expression.

\section{CENTER-OF-MASS KINETIC ENERGY SUBTRACTION}

Since the energy of a particle-antiparticle state in the presence of a magnetic field has a non-trivial dependence on the pseudomomentum quantum number $\mathbf{K}$, one has to specify the precise manner in which the energy associated with the COM motion is subtracted from the total energy. Our prescription for doing this is to subtract $\left\langle\mathbf{P}_{\text {kinetic }}\right\rangle^{2} / 2 M$ where $M=m_{1}+m_{2}=2 m_{q}$ from the total energy with $\left\langle\mathbf{P}_{\text {kinetic }}\right\rangle$ computed via Eq. (28).

As a concrete example, let's return to the case of a harmonic interaction. As demonstrated in the previous section this can be computed analytically in the case of a harmonic interaction. Taking Eq. (38) and subtracting $\left\langle\mathbf{P}_{\text {kinetic }}\right\rangle^{2} / 2 M$ with $\left\langle\mathbf{P}_{\text {kinetic }}\right\rangle$ given in Eq. (43) we obtain

$$
\begin{aligned}
\tilde{E}_{\mathbf{K}, n_{\perp} n_{z} \ell} & =E_{\mathbf{K}, n_{\perp} n_{z} \ell}-\frac{\left\langle\mathbf{P}_{\text {kinetic }}\right\rangle^{2}}{2 M}, \\
& =\frac{2 \omega_{c}^{2} \omega_{0}^{2}\left(K_{x}^{2}+K_{y}^{2}\right)}{M\left(\omega_{c}^{2}+4 \omega_{0}^{2}\right)^{2}}+\left(n_{z}+\frac{1}{2}\right) \omega_{0}+\left(2 n_{\perp}+1+|\ell|\right) \sqrt{\omega_{0}^{2}+\frac{\omega_{c}^{2}}{4}}-\boldsymbol{\mu} \cdot \mathbf{B}+M .
\end{aligned}
$$

As we can see from this expression, as $B \rightarrow 0$ the dependence of the COM-subtracted energy on the COM pseudomomentum vanishes as it should; however, for non-vanishing background magnetic field, there is still a residual dependence on the components of the pseudomomentum which are perpendicular to the background magnetic field. In the case of the harmonic interaction, we are able to obtain the answer analytically. In cases other than the simple harmonic interaction, it may not be possible to obtain analytic expressions. Absent analytic expressions for the energy and necessary expectation values, one must perform the subtraction prescribed in this section numerically. 


\section{QUARKONIUM SPIN-MIXING}

Thus far we have not discussed the effects of the magnetic field-spin coupling for particleantiparticle states. In this respect states like the $J / \psi$ and $\Upsilon$ are similar to positronium (see e.g. [80, 81] and references therein). We now review the mixing of the single and triplet states for completeness. The Hamiltonian can be written in the form

$$
\hat{H}=\hat{H}_{0}-\boldsymbol{\mu} \cdot \mathbf{B},
$$

where $\hat{H}_{0}$ collects all terms which depend on the spatial coordinates and

$$
\begin{aligned}
\boldsymbol{\mu} & =\boldsymbol{\mu}_{q}+\boldsymbol{\mu}_{\bar{q}} \\
& =g^{-} \mu_{q} \mathbf{S}_{q}+g^{+} \mu_{q} \mathbf{S}_{\bar{q}} \\
& =\frac{1}{2} g^{\prime} \mu_{q}\left(\boldsymbol{\sigma}^{-}-\boldsymbol{\sigma}^{+}\right),
\end{aligned}
$$

where $\mu_{q}=Q / 2 m_{q}$ is the quark magneton and in going from the second to third lines we have used $g^{-}=-g^{+}=g^{\prime}$. Herein, we ignore effects of the anomalous magnetic moment and take $g^{\prime}=2$. The coupled spin states to be considered are

$$
\begin{aligned}
|11\rangle & =|\uparrow \uparrow\rangle, \\
|1-1\rangle & =|\downarrow \downarrow\rangle, \\
|10\rangle & =\frac{1}{\sqrt{2}}(|\uparrow \downarrow\rangle+|\downarrow \uparrow\rangle), \\
|00\rangle & =\frac{1}{\sqrt{2}}(|\uparrow \downarrow\rangle-|\downarrow \uparrow\rangle) .
\end{aligned}
$$

In the case of $c \bar{c}$ states, the $1 \mathrm{~s}$ triplet and singlet states correspond to the $J / \psi$ and the $\eta_{c}$, respectively. For $b \bar{b}$ states the 1s triplet and singlet states correspond to the $\Upsilon(1 s)$ and $\eta_{b}$, respectively. Without a spin-spin interaction, these states would be degenerate. With a spin-spin interaction, the triplet and single states split. In vacuum, the charmonium $1 \mathrm{~s}$ splitting is approximately $\Delta E=113 \mathrm{MeV}$ and for bottomonium it is approximately $\Delta E=$ $63 \mathrm{MeV}$.

In the presence of a magnetic field there is mixing between some of these spin states. One can easily verify that

$$
\begin{aligned}
\left(\sigma_{z}^{+}-\sigma_{z}^{-}\right)|1 \pm 1\rangle & =0, \\
\left(\sigma_{z}^{+}-\sigma_{z}^{-}\right)|10\rangle & =2|00\rangle, \\
\left(\sigma_{z}^{+}-\sigma_{z}^{-}\right)|00\rangle & =2|10\rangle .
\end{aligned}
$$


From this we see that there is no magnetic field effect on the $|1 \pm 1\rangle$ spin states but there will be mixing between the $|00\rangle$ and $|10\rangle$ spin states. To determine the effect of the mixing we need only consider the two-dimensional eigensystem for the $|00\rangle$ and $|01\rangle$ states. To proceed we shift the zero of the Hamiltonian energy to the midpoint between the unperturbed singlet and triplet states and write an effective Hamiltonian of the form

$$
H_{\mathrm{eff}}=\frac{\Delta E}{2}\left(\begin{array}{cc}
1 & \chi \\
\chi & -1
\end{array}\right)
$$

where

$$
\chi=\frac{2 g^{\prime} \mu_{q} B}{\Delta E} .
$$

The resulting eigenstates can be expressed as

$$
\left|\psi_{ \pm}\right\rangle=\frac{1}{\sqrt{1+\varepsilon_{ \pm}^{2}}}\left(|00\rangle+\varepsilon_{ \pm}|10\rangle\right)
$$

with $\varepsilon_{ \pm} \equiv\left(1 \pm \sqrt{1+\chi^{2}}\right) / \chi$. One can verify that the states are orthogonal and normalized. The energy shifts of the states relative to the case of no spin-magnetic field effects taken into account are

$$
\Delta E_{ \pm}= \pm \frac{\Delta E}{2}\left(\sqrt{1+\chi^{2}}-1\right) .
$$

As a result, we see an increase in the energy of the $|10\rangle$ state and a decrease in the energy of the $|00\rangle$ state. In what follows we will indicate the two degenerate unmixed triplet states with a superscript \pm , e.g. $J / \psi^{ \pm}$and $\Upsilon^{ \pm}$, and the spin-mixed triplet state with a superscript 0 , e.g. $J / \psi^{0}$ and $\Upsilon^{0}$. To close this section we note that in addition to the shifts in the energy levels, the state-mixing implies that e.g. some portion of $|10\rangle$ decays will be suppressed, instead appearing as decays with an invariant mass given by the energy of the $\left|\psi_{-}\right\rangle$state. This will cause suppression of e.g. $\Upsilon$ decays to lepton pairs and turn on decays of the $\eta_{b}$ to lepton pairs. This would manifest itself experimentally as a reduction in dilepton yields at the $\Upsilon$ mass along and the appearance of a peak at the mass of the $\eta_{b}$. The suppression described above is similar to the experimentally well-known magnetic field suppression of the ortho-positronium $3 \gamma$ decays [74, 75, 77].

\section{HAMILTONIAN REDUCTION AND CHOICE OF POTENTIAL}

In some cases, such as the case of a harmonic interaction, the energies and wave functions can be solved for analytically; however, in most cases this is not possible. In these cases 
it is necessary to solve the Schrödinger equation numerically. In practice, we can subtract out any terms which are independent of the position from Eq. (24). In addition, if the potential still possesses azimuthal symmetry we can set either $K_{x}$ or $K_{y}$ to zero by rotating the coordinate system appropriately. We choose herein to set $K_{y}$ to zero. The resulting Hamiltonian which is used in the numerical solutions is then of the form

$$
\mathcal{H}_{\text {rel }}^{\prime}=-\frac{\nabla^{2}}{2 \mu}+\frac{q B}{4 \mu} K_{x} y+\frac{q^{2} B^{2}}{8 \mu} \rho^{2}+V(\mathbf{r}) .
$$

After numerical solution using (54) the constant terms can be added back in manually in order to obtain the full energy eigenvalues.

For the charmonium and bottomonium states considered in this manuscript we use a Cornell potential plus a spin-spin interaction with a separate spin-spin potential

$$
V(r)=-\frac{4}{3} \frac{\alpha_{s}}{r}+\sigma r+\left(\mathbf{S}_{1} \cdot \mathbf{S}_{2}\right) V_{s}(r) .
$$

The expectation value $\left\langle\mathbf{S}_{1} \cdot \mathbf{S}_{2}\right\rangle$ reduces to $-3 / 4$ for the singlet state and $1 / 4$ for the triplet states. For the spin potential $V_{s}(r)$ we use a form found from fits to the charm spin-spin potential in lattice studies 82

$$
V_{s}(r)=\gamma e^{-\beta r}
$$

For charmonia, the constants $\gamma$ and $\beta$ above were fit to lattice data in Ref. [82]. They found $\gamma=0.825 \mathrm{GeV}$ and $\beta=1.982 \mathrm{GeV}$. In this paper we allow for variation of $\gamma$. For both charm and bottom states we will hold $\beta$ fixed to the value from Ref. [82, but we adjust the amplitude $\gamma$ in order to reproduce the experimentally measured splittings using Eq. (55) as the interaction potential. We present the resulting parameter sets and the corresponding $B=0$ spectra of charmonium and bottomonium states in App. A. For the bottom system we present a single "tuning" which reproduces all states through the $\Upsilon(3 s)$ with a maximum error of $0.22 \%$. In the charm sector, we consider two different tunings: (a) the bottomtuned parameter set just described (see Tables I and II in App. A) and (b) a charm-tuned parameter set which reproduces the masses of the $c \bar{c} 1 s$ and $2 s$ states with a maximum error of $1.3 \%$ (see Table III in App. A).

We note that the interaction potential and the non-derivative terms in (54) can be combined into a "pseudopotential" of the form

$$
V_{\text {pseudo }}(r)=\frac{q B}{4 \mu} K_{x} y+\frac{q^{2} B^{2}}{8 \mu} \rho^{2}-\frac{4}{3} \frac{\alpha_{s}}{r}+\sigma r+\left(\mathbf{S}_{1} \cdot \mathbf{S}_{2}\right) \gamma e^{-\beta r} .
$$


(a)

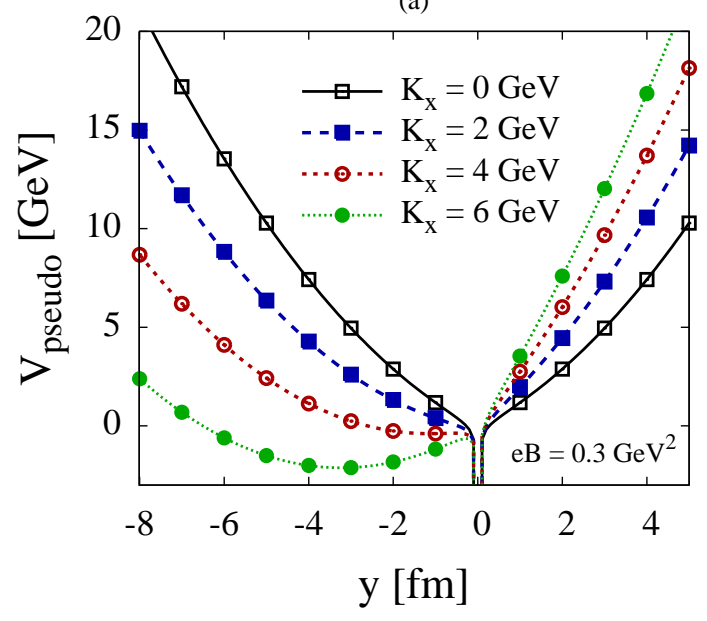

(b)

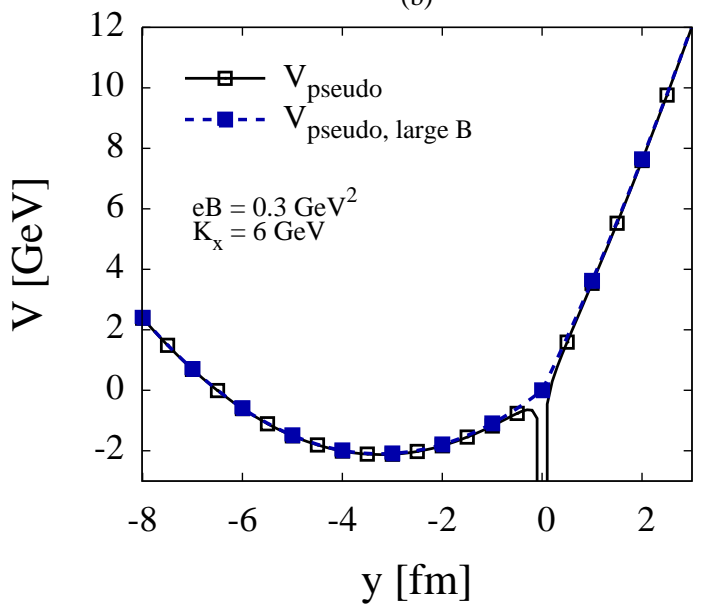

FIG. 1. (a) The pseudopotential (57) as a function of $y$ with $x=z=0$ for charmonium states using the parameters listed in App. A Table II. The magnetic field amplitude is assumed to be $e B=0.3 \mathrm{GeV}^{2}$ and we take $K_{x} \in\{0,2,4,6\} \mathrm{GeV}$. (b) Comparison of the exact pseudopotential (57) with the approximate form $(58)$ for $e B=0.3 \mathrm{GeV}^{2}$ and $K_{x}=6 \mathrm{GeV}$.

In Fig. 1(a) we plot the pseudopotential (57) as a function of $y$ with $x=z=0$ for charmonium states using the parameters listed in App. A Table II. The magnetic field amplitude is assumed to be $e B=0.3 \mathrm{GeV}^{2}$ and we take $K_{x} \in\{0,2,4,6\} \mathrm{GeV}$. As can be seen from this figure, at large magnetic field magnitude it is possible for the potential to develop a non-trivial minimum which for positive $K_{x}$ is at negative $y$. This minimum is related to so-called motional Stark effect which was originally discussed in [44] (see [47] for a discussion in the context of positronium) and recently discussed in the context of quarkonium in Ref. [60]. As a result of this minimum, for large $e B$ and $K_{x}$ the wave function becomes bilocalized. For large enough $K_{x}$ the wavefunction will be dominated by the leftmost minimum and the state will be "ionized" by magnetic field; however, we note that this state is, strictly speaking, not a free state since it is still confined in space by the magnetic field.

We note, for later use, that for large $q B, K_{x}$, and $r$, one can ignore the third and fifth terms in (57) to good approximation. Doing this and setting $x=z=0$ one obtains

$$
V_{\text {pseudo, large } B}(x=0, y, z=0) \simeq \frac{q B}{4 \mu} K_{x} y+\frac{q^{2} B^{2}}{8 \mu} y^{2}+\sigma|y| .
$$

We compare this approximate form to the exact pseudopotential in Fig. 1(b) for the case of charm quarks which have charge $q=2 e / 3$. Based on this expression we can find the 
approximate location of the leftmost minimum

$$
y_{\min } \simeq \frac{4 \sigma \mu-q B K_{x}}{q^{2} B^{2}}
$$

from which we learn that for $q B K_{x} \gtrsim 4 \sigma \mu$ there is a non-trivial minimum at negative

$y 2^{2}$ For charmonium (using the parameters listed in App. A Table III), this translates to the condition $e B K_{x} \gtrsim 0.673 \mathrm{GeV}^{3}$ and for bottomonium (using the parameters listed in App. A Table If $e B K_{x} \gtrsim 5.92 \mathrm{GeV}^{3}$. For the maximum magnetic field of $e B=0.3 \mathrm{GeV}^{2}$ considered herein this translates into the constraint $K_{x} \gtrsim 2.24 \mathrm{GeV}$ and $K_{x} \gtrsim 19.7 \mathrm{GeV}$ for charmonium and bottomonium, respectively. For $K_{x}$ larger than these thresholds, the state becomes bilocalized and eventually falls into the "harmonic" well. At this point the state is no longer bound by particle-anti-particle interactions, but is instead localized in space by the magnetic field.

In terms of practicalities for the numerics, we note that we use the approximate value in Eq. (59) to shift the potential along the $y$ direction for large values $K_{x}$ in order to obtain more accurate numerical results without having to resort to large volumes and/or anisotropic lattices.

\section{RESULTS}

We now present our results using the pseudopotential (57) for both charmonium and bottomonium states. For the bottomonium states, the potential parameters and resulting vacuum spectra are listed in App. A Table I. For charmonium states, the potential parameters and resulting vacuum spectra are listed in App. A Table III. The numerical algorithm used to find the eigenfunctions and eigenvalues is described in App. B. We note that we have tested the numerical algorithm using a harmonic interaction and have found agreement between the extracted wave functions, energy eigenvalues, etc. and the analytic formulae presented in previous sections to within machine precision. This gives us confidence in our numerical method.

\footnotetext{
${ }^{2}$ If $q$ is negative, the potential minimum appears at positive $y$ instead.
} 
(a)

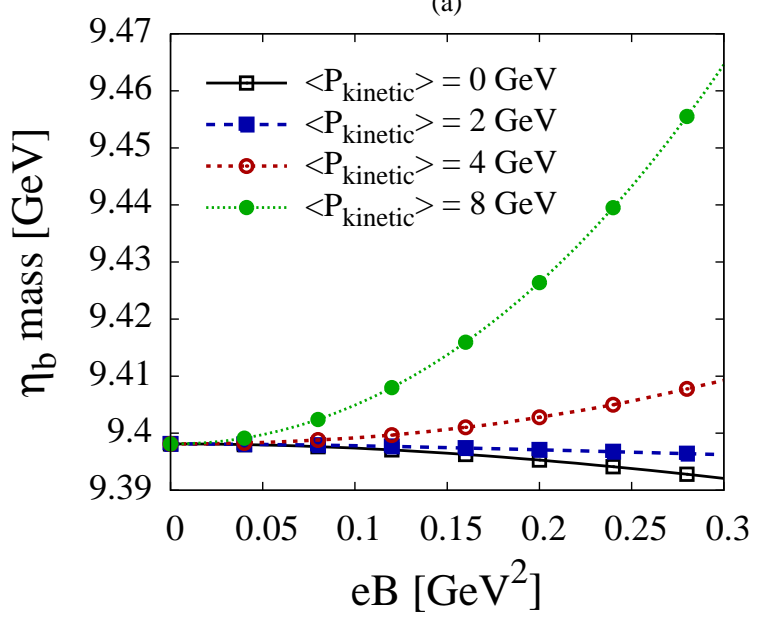

(b)

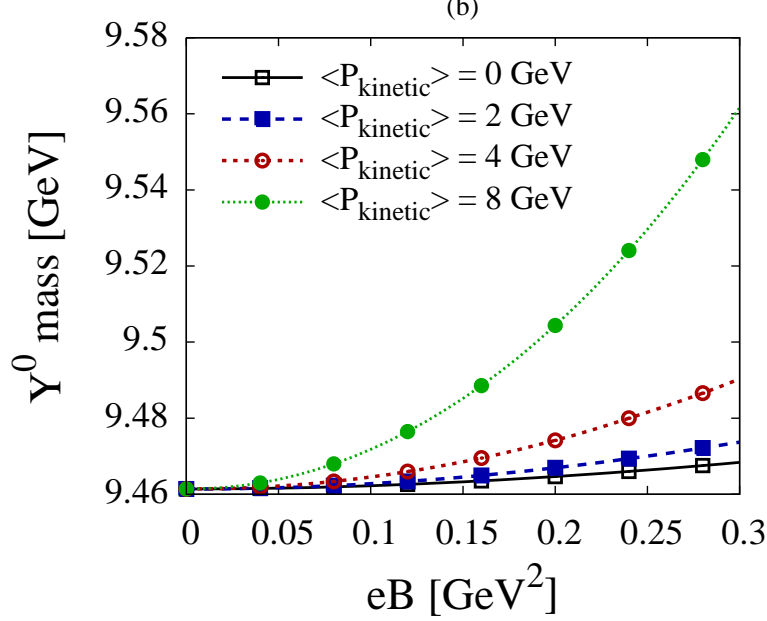

(c)

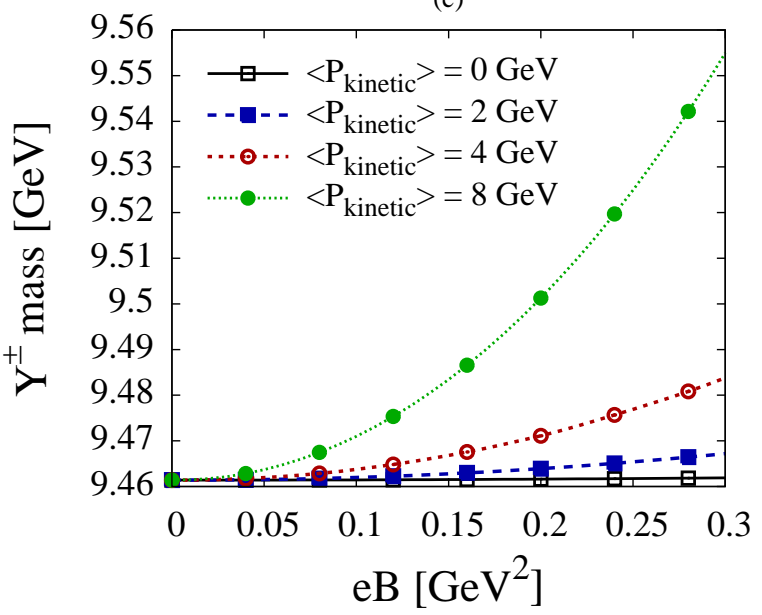

FIG. 2. Masses of the (a) $\eta_{b}$, (b) $\Upsilon^{0}$, and (c) $\Upsilon^{ \pm}$as a function of $e B$ for $\left\langle P_{\text {kinetic }}\right\rangle \in\{0,2,4,8\}$ $\mathrm{GeV}$.

\section{A. Bottomonia}

We first consider bottomium states. In Fig. 2 we plot the masses of the (a) $\eta_{b}$, (b) $\Upsilon^{0}$, and (c) $\Upsilon^{ \pm}$as a function of $e B$ for $\left\langle P_{\text {kinetic }}\right\rangle \in\{0,2,4,8\} \mathrm{GeV}$. For $\left\langle P_{\text {kinetic }}\right\rangle=0 \mathrm{GeV}$, we see the pattern expected, namely that the $\eta_{b}$ mass is lowered due to spin-mixing, the $\Upsilon^{0}$ mass increases for the same reason, and the $\Upsilon^{ \pm}$states are very-weakly affected (there is a small change in the mass due to the magnetic potential effects, but it is negligible). As we increase $\left\langle P_{\text {kinetic }}\right\rangle$, we see that the masses of all states increase. The result is in agreement with what we obtained analytically for the harmonic interaction (see first term in Eq. (45)). For $\left\langle P_{\text {kinetic }}\right\rangle=0$ and $e B=0.3 \mathrm{GeV}^{2}$ one sees a $0.06 \%$ decrease in the mass of the $\eta_{b}$. For $\left\langle P_{\text {kinetic }}\right\rangle=8 \mathrm{GeV}$, one sees an increase of $0.71 \%$ in the $\eta_{b}$ mass. For the $\Upsilon$ states, the mass 


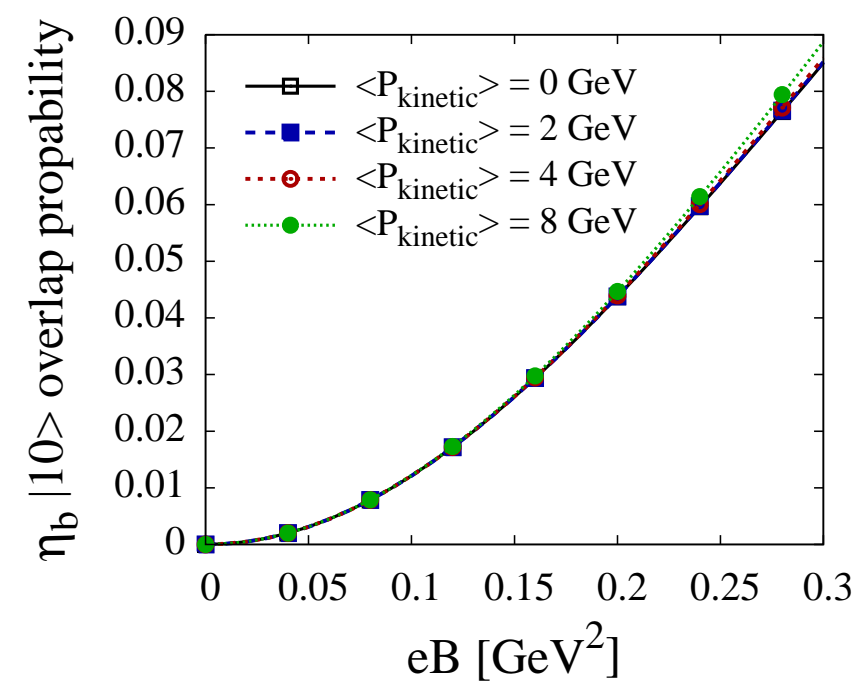

FIG. 3. Probability of finding $|10\rangle$ in the $\eta_{b}$ state as a function of $e B$ for $\left\langle P_{\text {kinetic }}\right\rangle \in\{0,2,4,8\}$ $\mathrm{GeV}$.

is a monotonically increasing function of $e B$ and $\left\langle P_{\text {kinetic }}\right\rangle$. The maximum mass increase is on the order of $1.1 \%$ for the $\Upsilon$ states.

Based on the findings above one can estimate the effect of strong magnetic fields on bottomonium production in the LHC heavy ion collisions $\left(e B \sim 0.3 \mathrm{GeV}^{2}\right)$. The cross sections for quarkonium production from both gluon-gluon fusion and quark-antiquark annihilation both scale (to leading order) as $M^{-2}$. Assuming that we need only build in the mass correction in order to account for the magnetic field, the maximal effect on 1s bottomonium states can be estimated to be on the order of a $2 \%$ effect.

We can extract the energy difference between the singlet and triplet states to determine the overlap probability for the $|10\rangle$ (triplet) state with, e.g. the $\eta_{b}$ state, via Eq. (52). In vacuum, the $\eta_{b}$ is a pure singlet state, however, a background magnetic field causes a mixing of the singlet and triplet states. In Fig. 3 we plot the $\eta_{b}$ triplet overlap probability as a function of $e B$ for $\left\langle P_{\text {kinetic }}\right\rangle \in\{0,2,4,8\} \mathrm{GeV}$. As we can see from this figure, at LHC energies one estimates the overlap probability to be approximately $8.5 \%$. This percentage of $\eta_{b}$ states would be able to decay through dilepton decay. Correspondingly, there would be an $8.5 \%$ reduction in the dilepton decays from the $\Upsilon^{0}$ state. The $\Upsilon^{ \pm}$states do not mix and would not have their dilepton decay rate modified. Averaging over the three different types of $\Upsilon$ states we would predict an approximately $2.8 \%$ suppression of $\Upsilon(1 s)$ decays. 
(a)

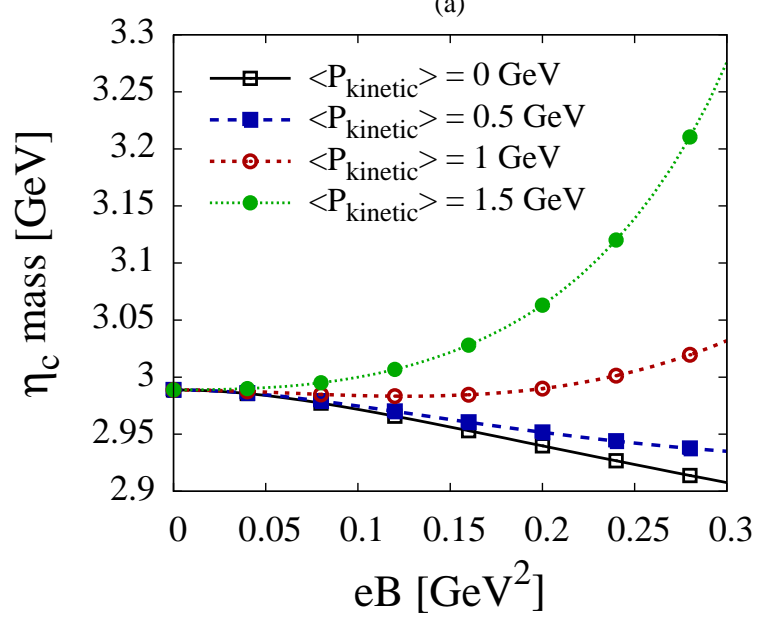

(b)

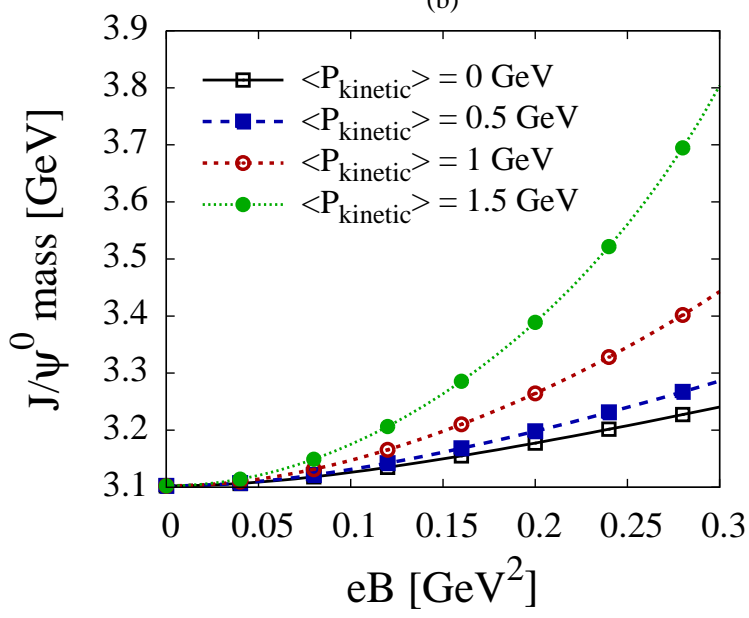

(c)

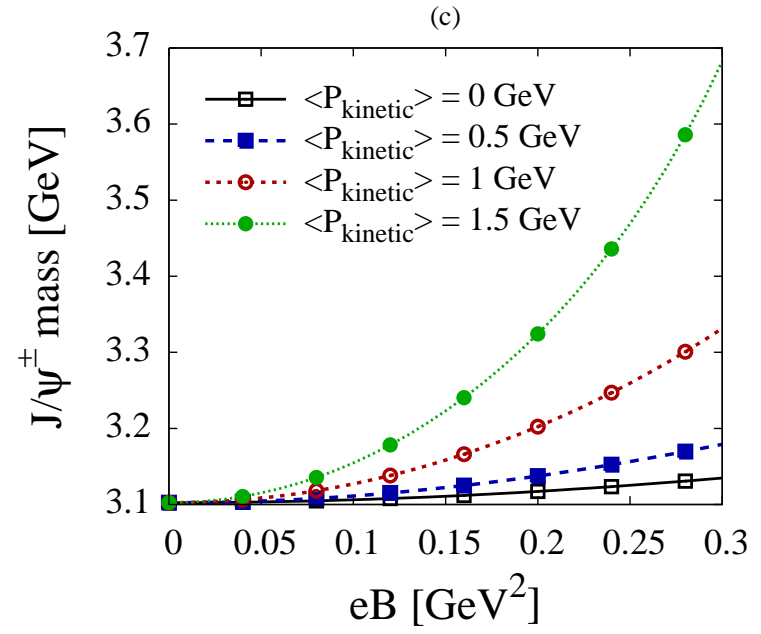

FIG. 4. Masses of the (a) $\eta_{c}$, (b) $J / \psi^{0}$, and (c) $J / \psi^{ \pm}$as a function of $e B$ for $\left\langle P_{\text {kinetic }}\right\rangle \in\{0,0.5,1,2\}$ $\mathrm{GeV}$.

The dileptons which failed to come from the $\Upsilon^{0}$ decays, would instead appear at the mass of the $\eta_{b}$ state. This would manifest itself through a peak in the dilepton spectrum at the $\eta_{b}$ invariant mass. We note, however, that given finite detector resolution, it may not be possible to experimentally resolve this feature in the dilepton spectrum invariant mass spectrum. The splitting between the $\eta_{b}$ and $\Upsilon$ vacuum masses is approximately $63 \mathrm{MeV}$ and this is only weakly dependent on the magnetic field. The CMS and ALICE experiments have a invariant mass resolution on the order of $100 \mathrm{MeV}$ [83, 84] so they would not be able to see this effect, instead they would see a slight broadening of the $\Upsilon(1 s)$ peak. 


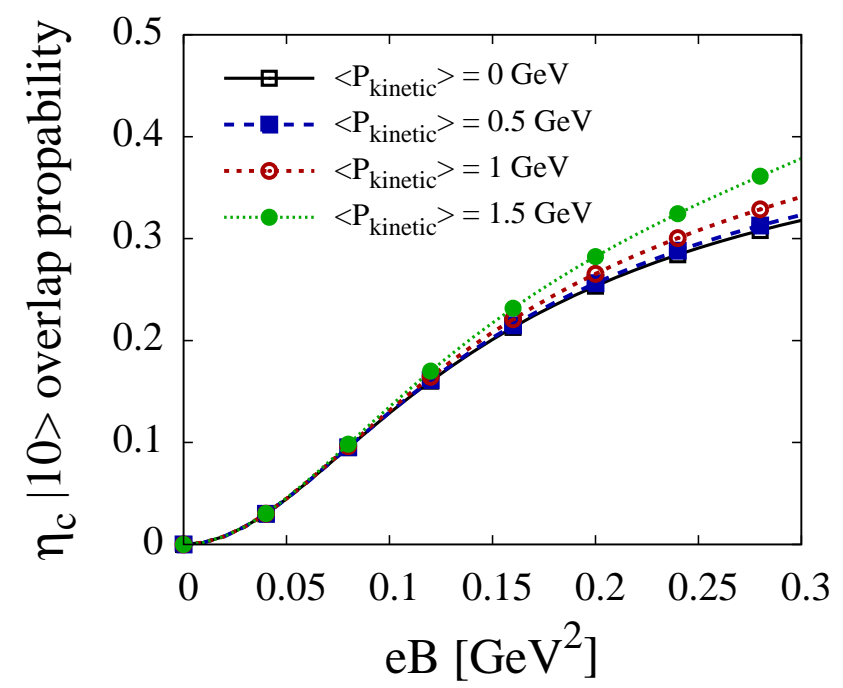

FIG. 5. Probability of finding $|10\rangle$ in the $\eta_{c}$ state as a function of $e B$ for $\left\langle P_{\text {kinetic }}\right\rangle \in\{0,0.5,1,2\}$ $\mathrm{GeV}$.

\section{B. Charmonia}

We now turn our attention to the charmonium states. In Fig. 4 we plot the masses of the (a) $\eta_{c}$, (b) $J / \psi^{0}$, and (c) $J / \psi^{ \pm}$as a function of $e B$ for $\left\langle P_{\text {kinetic }}\right\rangle \in\{0,0.5,1,1.5\} \mathrm{GeV}$ using the charmonium-tuned parameters listed in App. A Table III. $\mathrm{L}^{3}$

For $\left\langle P_{\text {kinetic }}\right\rangle=0 \mathrm{GeV}$, we see the pattern expected, namely that the $\eta_{c}$ mass is lowered due to spin-mixing, the $J / \psi^{0}$ mass increases for the same reason, and the $J / \psi^{ \pm}$states are weakly affected. For $K_{x}=0$ and $e B=0.3 \mathrm{GeV}^{2}$ one sees a $3.5 \%$ decrease in the mass of the $\eta_{c}$. For $K_{x}=1.5 \mathrm{GeV}$, one sees an increase of $19 \%$ in the $\eta_{c}$ mass. For the $J / \psi$ states, the mass is a monotonically increasing function of $e B$ and $\left\langle P_{\text {kinetic }}\right\rangle$. The maximum mass increase is on the order of $31 \%$ for the $J / \psi$ states. Again assuming that to leading order the $J / \psi$ production cross section scales like $M^{-2}$ one can estimate that this would result in a maximum suppression of $J / \psi$ my approximately $42 \%$, with the corresponding nuclear suppression being $R_{A A} \sim 0.58$.

In Fig. 5 we plot the $\eta_{c}$ triplet overlap probability as a function of $e B$ for $\left\langle P_{\text {kinetic }}\right\rangle \in$ $\{0,0.5,1,1.5\} \mathrm{GeV}$. As we can see from this figure, at LHC energies one estimates the overlap probability to be approximately $32 \%$. This percentage of $\eta_{c}$ states would be able

3 For a comparison of the results obtained using the bottom-tuned potential applied to charmonium states, see Fig. 6 in App. A and the surrounding discussion. 
to decay through dilepton decay. Correspondingly, there would be a $32 \%$ reduction in the dilepton decays from the $J / \psi^{0}$ state. The $J / \psi^{ \pm}$states do not mix and would not have their dilepton decay rate modified. Averaging over the three different types of $J / \psi$ states we would predict an approximately $11 \%$ suppression of $J / \psi$ decays. The dileptons which failed to come from the $J / \psi^{0}$ decays, would instead appear at the mass of the $\eta_{c}$ state. This would manifest itself through a peak in the dilepton spectrum at the $\eta_{c}$ invariant mass. Regarding the feasibility of measuring this effect experimentally, the splitting between the $\eta_{c}$ and $J / \psi$ vacuum masses is approximately $113 \mathrm{MeV}$ and the CMS and ALICE experiments have a invariant mass resolution on the order of $30 \mathrm{MeV}$ [83, 84]. As a result, it may be possible see hints of this effect in the charmonium sector. To truly confirm this effect, however, it would seem that either the detector resolution or the Crystal Ball function would need to be improved upon.

\section{CONCLUSIONS}

In this paper we have made a first investigation of the effects of an external magnetic field on charmonium and bottomonium states. We have taken into account the external potential associated with the magnetic field, motional effects, and the singlet-triplet mixing of states. We solved the resulting three-dimensional Schrödinger equation analytically for the case of a harmonic interaction and numerically for a realistic quarkonium potential consisting of a Cornell potential plus a spin-spin interaction. We demonstrated that it is not possible to fully factorize the Hamiltonian of the two-particle system in the presence of the magnetic field. Instead, one can introduce a conserved quantity called the pseudomomentum, $\mathbf{K}$, which allows one to write a compact "pseudopotential" for the system which has a nontrivial dependence on the components of $\mathbf{K}$ that are perpendicular to the magnetic field. We then derived a general relation between the pseudomomentum and the kinetic COM momentum of the system. For the harmonic interaction, the latter relation could be derived analytically for all states.

Herein we have considered states with COM momentum up to $1.5 \mathrm{GeV}$ in the case of 1s $J / \psi$ and $10 \mathrm{GeV}$ in the case of the $\Upsilon(1 s)$. For $J / \psi$ COM momentum larger than this threshold we find that the state will dissociate in the magnetic field (a similar conclusion but with a different threshold was found in Ref. [60]); however, since our results were derived in 
the context of a non-relativistic limit, one expects relativistic corrections to become quantitatively important at large momenta. For this reason, it seems necessary to reformulate the problem in a relativistic framework if one wants to arrive at more reliable conclusions about the phenomenological consequences on $J / \psi$ production. For $\Upsilon$ production, the threshold for magnetic field dissociation is estimated to be on the order of $20 \mathrm{GeV}$. At these high momenta, a relativistic treatment of the COM motion is necessary; however, for the range of $\Upsilon \mathrm{COM}$ momenta considered herein a non-relativistic treatment should be reasonable. Our results indicate that the maximal effect on $\Upsilon$ production is on the order of $2 \%$ and, as a result, it is probably safe to ignore this effect on theses states. For both systems, in order to minimize the effect of magnetic fields in experimental measurements of quarkonium suppression, one can apply transverse momentum cuts which eliminate states with high COM momentum.

As part of the analysis we presented a quantitative analysis of the effect of singlet-triplet spin mixing for both charmonium and bottomonium 1s states. The effect causes an increase in the mass of the $|10\rangle$ triplet state and a decrease in the mass of the $|00\rangle$ state. In addition, because of the mixing, some decays of the $|10\rangle$ will appear instead at the mass of the $|00\rangle$ state; however, given the fact that the splittings in the charmonium and bottomonium states are on the order of 113 and $62 \mathrm{MeV}$, respectively, it does not seem possible to use existing experimental configurations to fully resolve this effect. With limited resolution, the mixing would appear instead as a broadening of the triplet state peak.

The estimates of the phenomenological effect of static magnetic fields obtained herein are subject to two important caveats: (1) our investigations were restricted to the vacuum Cornell potential plus a spin-spin interaction and (2) we did not investigate the effect on excited states. Regarding caveat number one, in a future study we plan to include finitetemperature effects on the potential (see e.g. [85, 86]) and to simultaneously include more realistic vacuum potentials (see e.g. [87 91]). Since finite temperature effects reduce the binding energy and cause the states to be more extended in space, one can expect a priori that the magnetic field effect will be larger at finite temperature. Regarding caveat number two, we also plan a thorough investigation of magnetic field effects on excited states using realistic potential models. The effects on excited states are expected to be more important than on the ground state for two reasons: (a) excited states are more extended in space and are therefore more sensitive to the quadratic magnetic potential and (b) spin-mixing effects 
grow larger as the angular momentum representation of the state increases. Since excited state feed-down makes up on the order of $50 \%$ of both $J / \psi$ and $\Upsilon$ production one expects this to affect the ground states themselves.

Based on the two caveats laid out in the preceding paragraph, we expect that our estimates of the effect of static magnetic fields on heavy quarkonium production are a lower bound. That being said, one should also take into account the fact that the magnetic field generated in a heavy ion collision is neither static nor constant in space. One expects very strong magnetic fields only for the first 1-2 fm/c after the initial nuclear impact and as a result this would act to reduce the integrated magnetic field effect. In addition, it will be necessary to make a detailed investigation of the effect of magnetic field on the string tension and finite-temperature screened potential. We plan to investigate these effects in a future study. In closing, we have demonstrated in this paper that the effect of magnetic fields on heavy quarkonium, particularly the $J / \psi$, warrants further investigation. We have laid the ground work for such studies in the paper.

\section{ACKNOWLEDGEMENTS}

We thank F.S. Navarra and J. Noronha for motivation and useful discussions. J.A. was supported by DOE Grant No. DE-FG02-89ER40531. M.S. was supported in part by DOE Grant No. DE-SC0004104.

\section{Appendix A: Potential Tuning}

In this appendix we present comparisons of bottomonium and charmonium state masses computed using the model potential (55) and experimental data [92. We present results from the two different "tunings" which are used in the body of the manuscript separately.

\section{Bottom-tuned potential}

In Table I we compare bottomonia experimental data and the "bottom-tuned" potential model. The model results were computed on a lattice size of $256^{3}$ with lattice spacing of

$a=0.1 \mathrm{GeV}^{-1}$. The parameters used were $m_{b}=4.7 \mathrm{GeV}, \gamma=0.318 \mathrm{GeV}, \beta=1.982 \mathrm{GeV}$, 


\begin{tabular}{|c|c|c|c|c|}
\hline State & Name & Exp. 92 & Model & Rel. Err. \\
\hline $1^{1} S_{0}$ & $\eta_{b}(1 S)$ & $9.398 \mathrm{GeV}$ & $9.398 \mathrm{GeV}$ & $0.001 \%$ \\
\hline $1^{3} S_{1}$ & $\Upsilon(1 S)$ & $9.461 \mathrm{GeV}$ & $9.461 \mathrm{GeV}$ & $0.004 \%$ \\
\hline $1^{3} P_{0}$ & $\chi_{b 0}(1 P)$ & $9.859 \mathrm{GeV}$ & \multirow{4}{*}{$9.869 \mathrm{GeV}$} & \multirow{4}{*}{$0.21 \%$} \\
\hline $1^{3} P_{1}$ & $\chi_{b 1}(1 P)$ & $9.893 \mathrm{GeV}$ & & \\
\hline $1^{3} P_{2}$ & $\chi_{b 2}(1 P)$ & $9.912 \mathrm{GeV}$ & & \\
\hline $1^{1} P_{1}$ & $h_{b}(1 P)$ & $9.899 \mathrm{GeV}$ & & \\
\hline $2^{1} S_{0}$ & $\eta_{b}(2 S)$ & $9.999 \mathrm{GeV}$ & $9.977 \mathrm{GeV}$ & $0.22 \%$ \\
\hline $2^{3} S_{1}$ & $\Upsilon(2 S)$ & $10.002 \mathrm{GeV}$ & $9.999 \mathrm{GeV}$ & $0.03 \%$ \\
\hline $2^{3} P_{0}$ & $\chi_{b 0}(2 P)$ & $10.232 \mathrm{GeV}$ & \multirow{4}{*}{$10.246 \mathrm{GeV}$} & \multirow{4}{*}{$0.05 \%$} \\
\hline $2^{3} P_{1}$ & $\chi_{b 1}(2 P)$ & $10.255 \mathrm{GeV}$ & & \\
\hline $2^{3} P_{2}$ & $\chi_{b 2}(2 P)$ & $10.269 \mathrm{GeV}$ & & \\
\hline $2^{1} P_{1}$ & $h_{b}(2 P)$ & - & & \\
\hline $3^{1} S_{0}$ & $\eta_{b}(3 S)$ & - & $10.344 \mathrm{GeV}$ & - \\
\hline $3^{3} S_{1}$ & $\Upsilon(3 S)$ & $10.355 \mathrm{GeV}$ & $10.358 \mathrm{GeV}$ & $0.03 \%$ \\
\hline
\end{tabular}

TABLE I. Comparison of experimentally measured particle masses from Ref. [92 for the bottomonium system with "bottom-tuned" model predictions obtained using the potential model specified in Eq. (55). The parameters used were $m_{b}=4.7 \mathrm{GeV}, \gamma=0.318 \mathrm{GeV}, \beta=1.982 \mathrm{GeV}$, $\alpha_{s}=0.315443$, and $\sigma=0.210 \mathrm{GeV}^{2}$. In the case that there is no experimental data, we indicate this with a dash.

$\alpha_{s}=0.315443$, and $\sigma=0.210 \mathrm{GeV}^{2}$. Note that, since the potential model used herein does not include spin-orbit or tensor interactions, the model does not predict a splitting between the $\chi$ states. For these states, the error reported is computed from the average of the experimental masses.

In Table II we compare charmonia experimental data and the "bottom-tuned" potential model. The model results were computed on a lattice size of $256^{3}$ with lattice spacing of $a=0.2 \mathrm{GeV}^{-1}$. The parameters used were $m_{c}=1.29 \mathrm{GeV}, \gamma=0.825 \mathrm{GeV}, \beta=1.982 \mathrm{GeV}$, $\alpha_{s}=0.315443$, and $\sigma=0.210 \mathrm{GeV}^{2}$. 


\begin{tabular}{|c|c|c|c|c|}
\hline State & Name & Exp. [92] & Model & Rel. Error \\
\hline $1^{1} S_{0}$ & $\eta_{c}(1 S)$ & $2.984 \mathrm{GeV}$ & $3.048 \mathrm{GeV}$ & $2.2 \%$ \\
\hline $1^{3} S_{1}$ & $J / \psi(1 S)$ & $3.097 \mathrm{GeV}$ & $3.100 \mathrm{GeV}$ & $0.11 \%$ \\
\hline $2^{1} S_{0}$ & $\eta_{c}(2 S)$ & $3.639 \mathrm{GeV}$ & $3.721 \mathrm{GeV}$ & $2.3 \%$ \\
\hline $2^{3} S_{1}$ & $J / \psi(2 S)$ & $3.686 \mathrm{GeV}$ & $3.748 \mathrm{GeV}$ & $1.7 \%$ \\
\hline
\end{tabular}

TABLE II. Comparison of experimentally measured particle masses from Ref. [92] for the charmonium system with "bottom-tuned" model predictions obtained using the potential model specified in Eq. (55). The parameters used were $m_{c}=1.29 \mathrm{GeV}, \gamma=0.825 \mathrm{GeV}, \beta=1.982 \mathrm{GeV}$, $\alpha_{s}=0.315443$, and $\sigma=0.210 \mathrm{GeV}^{2}$.

\begin{tabular}{|c|c|c|c|c|}
\hline State & Name & Exp. [92] & Model & Rel. Error \\
\hline $1^{1} S_{0}$ & $\eta_{c}(1 S)$ & $2.984 \mathrm{GeV}$ & $2.989 \mathrm{GeV}$ & $0.16 \%$ \\
\hline $1^{3} S_{1}$ & $J / \psi(1 S)$ & $3.097 \mathrm{GeV}$ & $3.102 \mathrm{GeV}$ & $0.17 \%$ \\
\hline $2^{1} S_{0}$ & $\eta_{c}(2 S)$ & $3.639 \mathrm{GeV}$ & $3.590 \mathrm{GeV}$ & $1.3 \%$ \\
\hline $2^{3} S_{1}$ & $J / \psi(2 S)$ & $3.686 \mathrm{GeV}$ & $3.650 \mathrm{GeV}$ & $0.97 \%$ \\
\hline
\end{tabular}

TABLE III. Comparison of experimentally measured particle masses from Ref. 92 for the charmonium system with "charm-tuned" model predictions obtained using the potential model specified in Eq. (55). The parameters used were $m_{c}=1.29 \mathrm{GeV}, \gamma=2.06 \mathrm{GeV}, \beta=1.982 \mathrm{GeV}, \alpha_{s}=0.234$, and $\sigma=0.174 \mathrm{GeV}^{2}$.

\section{Charm-tuned potential}

In Table II we present a second parameter tuning which better reproduces the energy levels of low-lying charmonium states. As can be seen from this table, even when tuned to the charmonium states, the relative errors of the heavy quark potential model spectra compared to experimental data are larger than those obtained for bottomonium states. This is to be expected and indicates that it is necessary to include relativistic corrections to obtain a more accurate reproduction of the spectrum of charmonium states. Comparing the relative errors of charmonia masses using the bottom-tuned and charm-tuned potential we expect that the charm-tuned potential is a better approximation than the bottom-tuned potential since the 
(a)

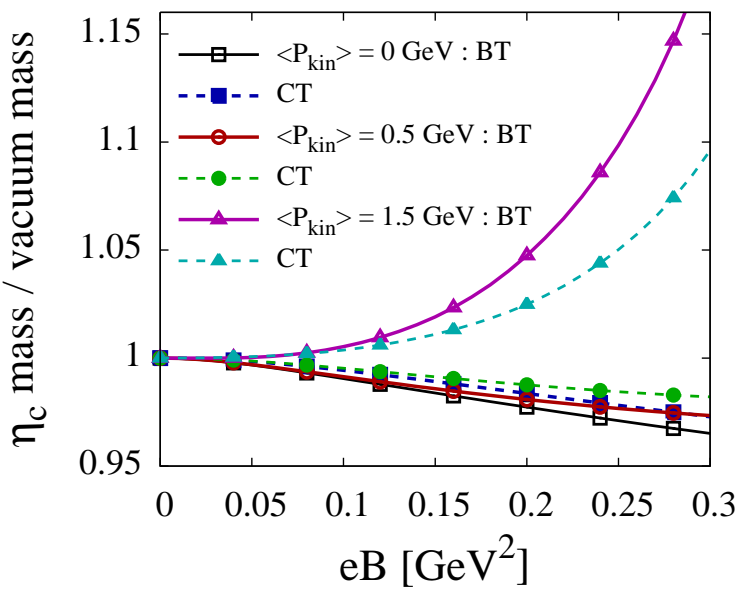

(c)

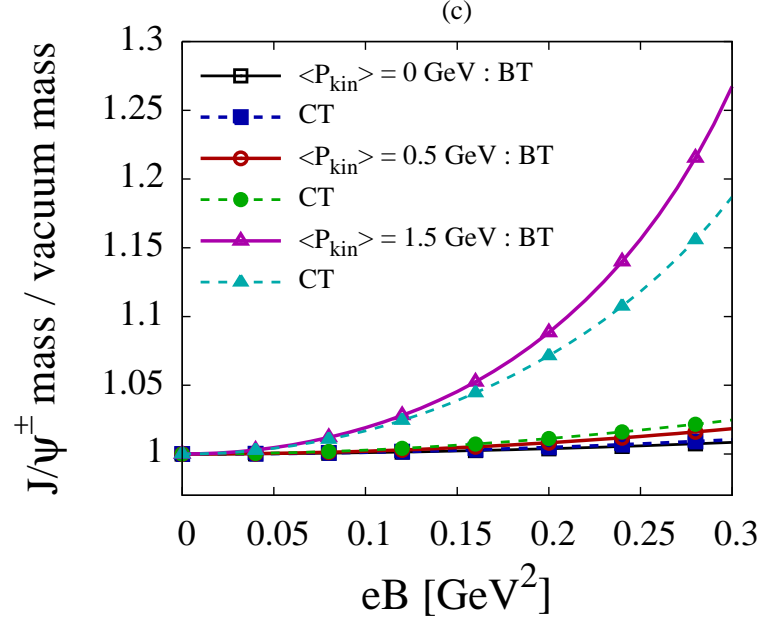

(b)

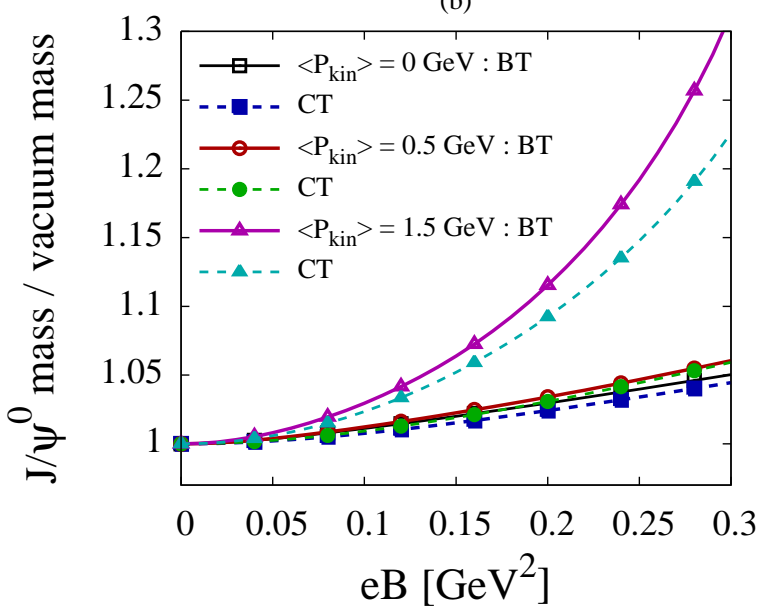

(d)

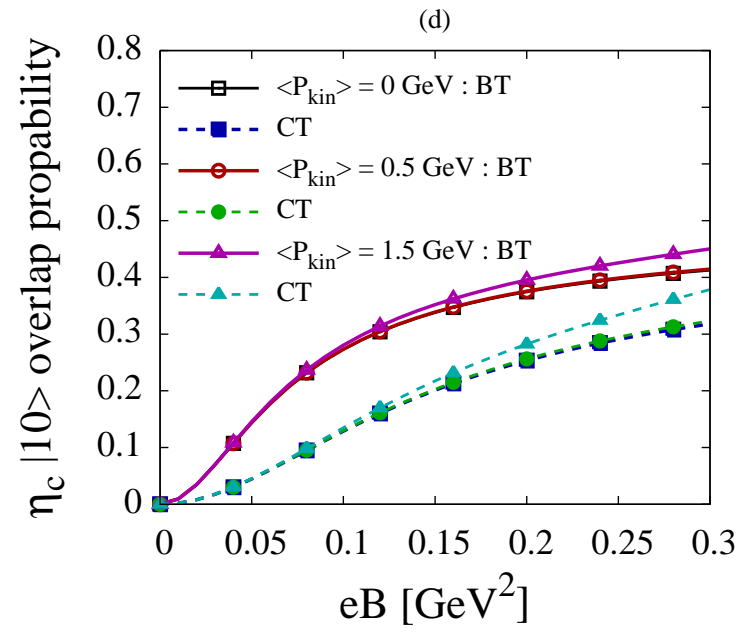

FIG. 6. Comparison of (a) $\eta_{c}$, (b) $J / \psi^{0}$, and (c) $J / \psi^{ \pm}$masses divided by the $e B=0$ vacuum masses and (d) triplet overlap probability as a function of $e B$ for $\left\langle P_{\text {kinetic }}\right\rangle \in\{0,0.5,1,1.5\} \mathrm{GeV}$. BT and CT indicate the results obtained using the bottom-tuned (Table II) and charm-tuned (Table III) potentials, respectively.

singlet-triplet split is very close to the experimentally determined splitting. That being said, we can use those two tunings to assess the dependence of our results on the assumed quark interaction potential. In Fig. 6 we show the scaled masses and triplet overlap probabilities using the two different tunings. In the figure, the bottom-tuned results are indicated by "BT" and the charm-tuned results by "CT". As we can see from this figure, the results obtained with the two different tunings are in qualitative agreement; however, we reiterate that we expect the charm-tuned results to be a better approximation. 


\section{Appendix B: Numerical Method}

To solve the resulting Schrödinger equation we use the finite difference time domain method [93 95]. Here we briefly review the technique. To determine the wave functions of bound quarkonium states, we must solve the time-independent Schrödinger equation for the relative wave function

$$
\hat{H}_{\mathrm{rel}} \Psi_{v}(\mathbf{r})=E_{v} \Psi_{v}(\mathbf{r}),
$$

on a three-dimensional lattice in coordinate space. The index $v$ on the eigenfunctions, $\phi_{v}$, and energies, $E_{v}$, represents a list of all relevant quantum numbers. To obtain the timeindependent eigenfunctions we start with the time-dependent Schrödinger equation

$$
i \frac{\partial}{\partial t} \Psi(\mathbf{x}, t)=\hat{H}_{\mathrm{rel}} \Psi(\mathbf{x}, t),
$$

which can be solved by expanding in terms of the eigenfunctions, $\Psi_{v}(\mathbf{r})$ :

$$
\Psi(\mathbf{r}, t)=\sum_{v} c_{v} \Psi_{v}(\mathbf{r}) e^{-i E_{v} t} .
$$

If one is only interested in the lowest energy states (ground state and first few excited states) an efficient way to proceed is to transform (B2) and $(\bar{B} 3)$ to Euclidean time using a Wick rotation, $\tau \equiv i t$ :

$$
\frac{\partial}{\partial \tau} \Psi(\mathbf{r}, \tau)=-\hat{H}_{\mathrm{rel}} \psi(\mathbf{r}, \tau),
$$

and

$$
\Psi(\mathbf{r}, \tau)=\sum_{v} c_{v} \Psi_{v}(\mathbf{r}) e^{-E_{v} \tau} .
$$

For details of the discretizations used etc. we refer the reader to Refs. 94].

\section{Finding the ground state}

By definition, the ground state is the state with the lowest energy eigenvalue, $E_{0}$. Therefore, at late imaginary time the sum over eigenfunctions $(\overline{B 5})$ is dominated by the ground state eigenfunction

$$
\lim _{\tau \rightarrow \infty} \Psi(\mathbf{r}, \tau) \rightarrow c_{0} \Psi_{0}(\mathbf{r}) e^{-E_{0} \tau} .
$$

Due to this, one can obtain the ground state wavefunction, $\phi_{0}$, and energy, $E_{0}$, by solving Eq. (B4) starting from a random three-dimensional wavefunction, $\Psi_{\text {initial }}(\mathbf{r}, 0)$, and evolving 
forward in imaginary time. The initial wavefunction should have a nonzero overlap with all eigenfunctions of the Hamiltonian; however, due to the damping of higher-energy eigenfunctions at sufficiently late imaginary times we are left with only the ground state, $\Psi_{0}(\mathbf{r})$. Once the ground state wavefunction (or any other wavefunction) is found, we can compute its energy eigenvalue via

$$
E_{v}(\tau \rightarrow \infty)=\frac{\left\langle\Psi_{v}|\hat{H}| \Psi_{v}\right\rangle}{\left\langle\Psi_{v} \mid \Psi_{v}\right\rangle}=\frac{\int d^{3} \mathbf{x} \Psi_{v}^{*} \hat{H} \Psi_{v}}{\int d^{3} \mathbf{x} \Psi_{v}^{*} \Psi_{v}}
$$

\section{Finding the excited states}

The basic method for finding excited states is to first evolve the initially random wavefunction to large imaginary times, find the ground state wavefunction, $\Psi_{0}$, and then project this state out from the initial wavefunction and re-evolve the partial-differential equation in imaginary time. However, there are (at least) two more efficient ways to accomplish this. The first is to record snapshots of the $3 \mathrm{~d}$ wavefunction at a specified interval $\tau_{\text {snapshot }}$ during a single evolution in $\tau$. After having obtained the ground state wavefunction, one can go back and extract the excited states by projecting out the ground state wavefunction from the recorded snapshots of $\Psi(\mathbf{r}, \tau)$ [93, 94].

An alternative way to select different excited states is to impose a symmetry condition on the initially random wavefunction which cannot be broken by the Hamiltonian evolution [94. For example, one can select the first p-wave excited state by anti-symmetrizing the initial wavefunction around either the $x, y$, or $z$ axes. In the non-spherical case this method can be used to separate the different excited state polarizations in the quarkonium system and to determine their energy eigenvalues with high precision.

\section{Appendix C: Application of the sudden approximation}

In this appendix we explore what happens to a system which suddenly has a magnetic field turned on. We will model this as being instantaneous in order to simplify the treatment and restrict our attention to a $3 \mathrm{~d}$ harmonic oscillator eigenstate since it is possible to make much more analytic progress in this case. We start by positing that for $t<0$ there is no magnetic field and that the system is subject only to an internal harmonic interaction in which case the full state can be decomposed in terms of the no-magnetic-field eigenstates 
$\Phi_{k}^{(0)}$

$$
\Phi(t)=\sum_{k} c_{k} \Phi_{k}^{(0)} e^{-i E_{k}^{(0)} t} \quad t<0
$$

where $k$ collects all relevant quantum numbers and the sum represents a sum over discrete quantum numbers and integral for continuous quantum numbers. For $t \geq 0$ we can expand in terms of the eigenstates in the presence of the magnetic field $\Phi_{m}^{(1)}$

$$
\Phi(t)=\sum_{m} d_{m} \Phi_{m}^{(1)} e^{-i E_{m}^{(1)} t} \quad t \geq 0
$$

At $t=0$ we match the coefficients which requires

$$
\sum_{m} d_{m} \Phi_{m}^{(1)}=\sum_{k} c_{k} \Phi_{k}^{(0)}
$$

Projecting with $\Phi_{n}^{(1)}$ and using their orthonormality we obtain

$$
d_{n}=\sum_{k} c_{k}\left\langle\Phi_{n}^{(1)} \mid \Phi_{k}^{(0)}\right\rangle
$$

\section{Pure state for $t<0$}

If the state for $t<0$ is a pure state with $c_{k}=\delta_{k m}$ we obtain

$$
d_{n}=\left\langle\Phi_{n}^{(1)} \mid \Phi_{m}^{(0)}\right\rangle
$$

We now turn to the computation of the overlap integrals necessary for the case at hand. The $t<0$ states are

$$
\Phi_{\mathbf{P}, n_{\perp}^{0} n_{z}^{0} \ell^{0}}^{(0)}(\mathbf{R}, \mathbf{r})=\mathcal{N}^{(0)} \rho^{\left|\ell^{0}\right|} e^{i \ell^{0} \phi} e^{-\frac{1}{2} \gamma^{2}\left(\rho^{2}+z^{2}\right)} H_{n_{z}^{0}}(\gamma z) L_{n_{\perp}^{0}}^{\left|\ell^{0}\right|}\left(\gamma^{2} \rho^{2}\right) e^{i \mathbf{P} \cdot \mathbf{R}}
$$

where

$$
\mathcal{N}^{(0)}=\frac{\gamma^{\left|\ell^{0}\right|+3 / 2}}{\sqrt{2^{n_{z}^{0}} \pi^{3 / 2}}} \sqrt{\frac{n_{\perp}^{0} !}{n_{z}^{0 !}\left(\left|\ell^{0}\right|+n_{\perp}^{0}\right) !}}
$$

and the $t \geq 0$ states are

$$
\Phi_{\mathbf{K}, n_{\perp} n_{z} \ell}^{(1)}(\mathbf{R}, \mathbf{r})=\mathcal{N}^{(1)} \tilde{\rho}^{|\ell|} e^{i \ell \tilde{\phi}} e^{-\frac{1}{2} \gamma^{2} z^{2}} e^{-\frac{1}{2} \alpha^{2} \tilde{\rho}^{2}} H_{n_{z}}(\gamma z) L_{n_{\perp}}^{|\ell|}\left(\alpha^{2} \tilde{\rho}^{2}\right) e^{i\left(\mathbf{K}-\frac{1}{2} q \mathbf{B} \times \mathbf{r}\right) \cdot \mathbf{R}}
$$


with

$$
\begin{aligned}
\omega_{c} & =\frac{q B}{\mu}, \\
\alpha^{2} & =\mu \sqrt{\omega_{0}^{2}+\frac{\omega_{c}^{2}}{4}}, \\
\gamma^{2} & =\mu \omega_{0}, \\
\tilde{\rho}^{2} & =\left(x-\lambda K_{y}\right)^{2}+\left(y+\lambda K_{x}\right)^{2}, \\
\tilde{\phi} & =\arctan \left(\frac{y+\lambda K_{x}}{x-\lambda K_{y}}\right), \\
\lambda & =\frac{\omega_{c}}{4 \mu\left(\omega_{0}^{2}+\omega_{c}^{2} / 4\right)},
\end{aligned}
$$

and

$$
\mathcal{N}^{(1)}=\frac{\alpha^{|\ell|+1} \gamma^{1 / 2}}{\sqrt{2^{n_{z}} \pi^{3 / 2}}} \sqrt{\frac{n_{\perp} !}{n_{z} !\left(|\ell|+n_{\perp}\right) !}} .
$$

The six-dimensional overlap integral in relative cylindrical coordinates becomes

$$
\begin{aligned}
d_{n}=\mathcal{N}^{(0)} \mathcal{N}^{(1)} \int_{0}^{\infty} \rho & d \rho \int_{0}^{2 \pi} d \phi \int_{-\infty}^{\infty} d z \int d^{3} \mathbf{R} \rho^{\left|\ell^{0}\right|} \tilde{\rho}^{|\ell|} e^{i\left(\ell^{0} \phi-\ell \tilde{\phi}\right)} e^{-\gamma^{2} z^{2}} e^{-\frac{1}{2}\left(\gamma^{2} \rho^{2}+\alpha^{2} \tilde{\rho}^{2}\right)} \\
& \times H_{n_{z}}(\gamma z) H_{n_{z}^{0}}(\gamma z) L_{n_{\perp}^{0}}^{\left|\ell^{0}\right|}\left(\gamma^{2} \rho^{2}\right) L_{n_{\perp}}^{|\ell|}\left(\alpha^{2} \tilde{\rho}^{2}\right) e^{i\left(\mathbf{P}-\mathbf{K}+\frac{1}{2} q \mathbf{B} \times \mathbf{r}\right) \cdot \mathbf{R}}
\end{aligned}
$$

Using $\frac{1}{2} q \mathbf{B} \times \mathbf{r}=\frac{1}{2} q B(-y, x, 0)=\frac{1}{2} q B \rho(-\sin \phi, \cos \phi, 0)$ and the orthonormality of the Hermite polynomials we can perform the $z$ and $\mathbf{Z}$ integrations. Using the exponential we can further perform the $\mathbf{X}$ and $\mathbf{Y}$ integrations. The remaining two integrals are evaluated in cartesian coordinates. The result is

$$
d_{n}=\tilde{\mathcal{N}}_{n m}\left(\frac{2}{|q| B}\right)^{2} \delta_{n_{z} n_{z}^{0}} \delta\left(P_{z}-K_{z}\right) \rho^{\left|\ell^{0}\right|} \tilde{\rho}^{|\ell|} e^{i\left(\ell^{0} \phi-\ell \tilde{\phi}\right)} e^{-\frac{1}{2}\left(\gamma^{2} \rho^{2}+\alpha^{2} \tilde{\rho}^{2}\right)} L_{n_{\perp}^{0}}^{\left|\ell^{0}\right|}\left(\gamma^{2} \rho^{2}\right) L_{n_{\perp}}^{|\ell|}\left(\alpha^{2} \tilde{\rho}^{2}\right),
$$

where

$$
\begin{aligned}
\tilde{\mathcal{N}}_{n m} & =(2 \pi)^{3} \mathcal{N}^{(0)} \mathcal{N}^{(1)} \sqrt{\pi} 2^{n_{z}} n_{z} ! / \gamma=2(2 \pi)^{2} \alpha^{|\ell|+1} \gamma^{\left|\ell^{0}\right|+1} \sqrt{\frac{n_{\perp}^{0} ! n_{\perp} !}{\left(\left|\ell^{0}\right|+n_{\perp}^{0}\right) !\left(|\ell|+n_{\perp}\right) !}} \\
\rho^{2} & =x^{2}+y^{2}=\left(\frac{2}{q B}\right)^{2}\left[\left(P_{x}-K_{x}\right)^{2}+\left(P_{y}-K_{y}\right)^{2}\right] \\
\phi & =\arctan \left(\frac{y}{x}\right)=\arctan \left(\frac{P_{x}-K_{x}}{K_{y}-P_{y}}\right) \\
\tilde{\rho}^{2} & =\left(\frac{2}{q B}\right)^{2}\left[\left(\beta K_{y}-P_{y}\right)^{2}+\left(P_{x}-\beta K_{x}\right)^{2}\right] \\
\tilde{\phi} & =\arctan \left(\frac{P_{x}-\beta K_{x}}{\beta K_{y}-P_{y}}\right)
\end{aligned}
$$


with

$$
\beta \equiv \frac{8 \omega_{0}^{2}+\omega_{c}^{2}}{8 \omega_{0}^{2}+2 \omega_{c}^{2}}
$$

which satisfies $\frac{1}{2} \leq \beta \leq 1$. Note that the above definitions only apply for the probability amplitude $d_{n}$. For $\tilde{\rho}$ and $\tilde{\phi}$ in the wavefunction, we need to use the definitions in Eq. C9.

\section{Gaussian Wave Packet as Initial Condition}

Let's consider that the initial condition is not a pure state but instead a Gaussian linear combination

$$
\Phi(t)=\sum_{k} c_{k} \Phi_{k}^{(0)} e^{-i E_{k}^{(0)} t}
$$

where $k=\left(\ell, k_{z}, k_{\perp}, \mathbf{P}\right)$. We will assume that the system is in a well-defined internal state $\left(\ell^{0}, n_{z}^{0}, n_{\perp}^{0}\right)$ but has a spread in COM momentum:

$$
c_{k}=\sqrt{\frac{8 \pi^{3 / 2}}{\sigma^{3}}} \delta_{\ell^{0} \ell} \delta_{n_{z}^{0} k_{z}} \delta_{n_{\perp}^{0} k_{\perp}} e^{-\left(\mathbf{P}-\mathbf{P}^{0}\right)^{2} /\left(2 \sigma^{2}\right)} .
$$

In this case the coefficient $d_{n}$ is more complicated:

$$
d_{n}=\sum_{m} c_{m}\left\langle\Phi_{n}^{(1)} \mid \Phi_{m}^{(0)}\right\rangle
$$

where we can use the pure state result obtained previously

$$
\left\langle\Phi_{n}^{(1)} \mid \Phi_{m}^{(0)}\right\rangle=\tilde{\mathcal{N}}_{n m}\left(\frac{2}{|q| B}\right)^{2} \delta_{n_{z} n_{z}^{0}} \delta\left(P_{z}-K_{z}\right) \rho^{\left|\ell^{0}\right|} \tilde{\rho}^{|\ell|} e^{i\left(\ell^{0} \phi-\ell \tilde{\phi}\right)} e^{-\frac{1}{2}\left(\gamma^{2} \rho^{2}+\alpha^{2} \tilde{\rho}^{2}\right)} L_{n_{\perp}^{0}}^{\left|\ell^{0}\right|}\left(\gamma^{2} \rho^{2}\right) L_{n_{\perp}}^{|\ell|}\left(\alpha^{2} \tilde{\rho}^{2}\right)
$$

with $m=\left(\ell^{0}, n_{z}^{0}, n_{\perp}^{0}, \mathbf{P}\right)$ and $n=\left(\ell, n_{z}, n_{\perp}, \mathbf{K}\right)$.

\section{Time evolution of the center-of-mass kinetic momentum}

We consider next the evolution of the COM kinetic momentum after the magnetic field is applied. We seek to evaluate $\left\langle\mathbf{P}_{\text {kinetic }}\right\rangle=\left\langle\Phi(t)\left|\mathbf{P}_{\text {kinetic }}\right| \Phi(t)\right\rangle$ for $t>0$.

$$
\left\langle\Phi(t)\left|\mathbf{P}_{\text {kinetic }}\right| \Phi(t)\right\rangle=\sum_{m, n} d_{m}^{*} d_{n}\left\langle\Phi_{m}^{(1)}\left|\mathbf{P}_{\text {kinetic }}\right| \Phi_{n}^{(1)}\right\rangle e^{-i\left(E_{n}^{(1)}-E_{m}^{(1)}\right) t}
$$


where $m=\left(\ell^{\prime}, n_{z}^{\prime}, n_{\perp}^{\prime}, \mathbf{K}^{\prime}\right), n=\left(\ell, n_{z}, n_{\perp}, \mathbf{K}\right)$, and

$$
\begin{gathered}
\sum_{m} \equiv \sum_{n_{z}^{\prime}=0}^{\infty} \sum_{\ell^{\prime}=-\infty}^{\infty} \sum_{n_{\perp}^{\prime}=0}^{\infty} \int \frac{d^{3} \mathbf{K}^{\prime}}{(2 \pi)^{3}} \\
\sum_{n} \equiv \sum_{n_{z}=0}^{\infty} \sum_{\ell=-\infty}^{\infty} \sum_{n_{\perp}=0}^{\infty} \int \frac{d^{3} \mathbf{K}}{(2 \pi)^{3}} \\
\left\langle\Phi_{m}^{(1)}\left|\mathbf{P}_{\text {kinetic }}\right| \Phi_{n}^{(1)}\right\rangle=\left\langle\Phi_{m}^{(1)}|\mathbf{K}-q \mathbf{B} \times \mathbf{r}| \Phi_{n}^{(1)}\right\rangle \\
=\mathbf{K} \delta_{m n}-q B\left\langle\Phi_{m}^{(1)}\left|\left(-\tilde{\rho} \sin \tilde{\phi}+\frac{c}{a}, \tilde{\rho} \cos \tilde{\phi}+\frac{b}{a}, 0\right)\right| \Phi_{n}^{(1)}\right\rangle,
\end{gathered}
$$

where $\delta_{m n}=\delta_{\ell^{\prime} \ell} \delta_{n_{z}^{\prime} n_{z}} \delta_{n_{\perp}^{\prime} n_{\perp}} \delta_{\mathbf{K}^{\prime} \mathbf{K}}, \delta_{\mathbf{K}^{\prime} \mathbf{K}} \equiv(2 \pi)^{3} \delta^{3}\left(\mathbf{K}^{\prime}-\mathbf{K}\right)$, and we remind the reader that $a=\mu\left(\omega_{0}^{2}+\omega_{c}^{2} / 4\right), b=\omega_{c} K_{y} / 4, c=\omega_{c} K_{x} / 4$. Considering the second term we have

$$
\left(-\left\langle\Phi_{m}^{(1)}|\tilde{\rho} \sin \tilde{\phi}| \Phi_{n}^{(1)}\right\rangle+\frac{c}{a} \delta_{m n},\left\langle\Phi_{m}^{(1)}|\tilde{\rho} \cos \tilde{\phi}| \Phi_{n}^{(1)}\right\rangle+\frac{b}{a} \delta_{m n}, 0\right) .
$$

To proceed, we first consider

$$
J_{m n}^{+} \equiv\left\langle\Phi_{m}^{(1)}\left|\tilde{\rho} e^{i \tilde{\phi}}\right| \Phi_{n}^{(1)}\right\rangle
$$

and

$$
J_{m n}^{-} \equiv\left\langle\Phi_{m}^{(1)}\left|\tilde{\rho} e^{-i \tilde{\phi}}\right| \Phi_{n}^{(1)}\right\rangle
$$

For $\ell \geq 0$

$$
J_{m n}^{+}=\frac{\delta_{\mathbf{K}^{\prime} \mathbf{K}} \delta_{n_{z}^{\prime} n_{z}} \delta_{\ell^{\prime}, \ell+1}}{\alpha}\left[\delta_{n_{\perp}^{\prime} n_{\perp}} \sqrt{n_{\perp}+\ell+1}-\delta_{n_{\perp}^{\prime}, n_{\perp}-1} \sqrt{n_{\perp}}\right] .
$$

For $\ell \leq-1$

$$
J_{m n}^{+}=\frac{\delta_{\mathbf{K}^{\prime} \mathbf{K}} \delta_{n_{z}^{\prime} n_{z}} \delta_{\ell^{\prime}, \ell+1}}{\alpha}\left[\delta_{n_{\perp}^{\prime} n_{\perp}} \sqrt{n_{\perp}-\ell}-\delta_{n_{\perp}^{\prime}, n_{\perp}+1} \sqrt{n_{\perp}+1}\right] .
$$

For $\ell \geq 1$

$$
J_{m n}^{-}=\frac{\delta_{\mathbf{K}^{\prime} \mathbf{K}} \delta_{n_{z}^{\prime} n_{z}} \delta_{\ell^{\prime}, \ell-1}}{\alpha}\left[\delta_{n_{\perp}^{\prime} n_{\perp}} \sqrt{n_{\perp}+\ell}-\delta_{n_{\perp}^{\prime}, n_{\perp}+1} \sqrt{n_{\perp}+1}\right] .
$$

For $\ell \leq 0$

$$
J_{m n}^{-}=\frac{\delta_{\mathbf{K}^{\prime} \mathbf{K}} \delta_{n_{z}^{\prime} n_{z}} \delta_{\ell^{\prime}, \ell-1}}{\alpha}\left[\delta_{n_{\perp}^{\prime} n_{\perp}} \sqrt{n_{\perp}-\ell+1}-\delta_{n_{\perp}^{\prime}, n_{\perp}-1} \sqrt{n_{\perp}}\right] .
$$

With these we have determined

$$
\begin{aligned}
\left\langle\Phi_{m}^{(1)}|\tilde{\rho} \sin \tilde{\phi}| \Phi_{n}^{(1)}\right\rangle & =\frac{1}{2 i}\left(J_{m n}^{+}-J_{m n}^{-}\right) \equiv \mathcal{S}_{m n}, \\
\left\langle\Phi_{m}^{(1)}|\tilde{\rho} \cos \tilde{\phi}| \Phi_{n}^{(1)}\right\rangle & =\frac{1}{2}\left(J_{m n}^{+}+J_{m n}^{-}\right) \equiv \mathcal{C}_{m n} .
\end{aligned}
$$


To evaluate $\left\langle\Phi(t)\left|\mathbf{P}_{\text {kinetic }}\right| \Phi(t)\right\rangle$ we will need

$$
\begin{aligned}
& \sum_{\ell=-\infty}^{\infty} \sum_{n_{\perp}=0}^{\infty} \sum_{\ell^{\prime}=-\infty}^{\infty} \sum_{n_{\perp}^{\prime}=0}^{\infty} \int_{\mathbf{K}^{\prime}} d_{m}^{*} d_{n} J_{m n}^{ \pm} e^{-i\left(E_{n}^{(1)}-E_{m}^{(1)}\right) t} \\
& =\frac{1}{\alpha} \sum_{\ell=0}^{\infty} \sum_{n_{\perp}=0}^{\infty}\left[d_{ \pm(\ell+1), n_{\perp}}^{*} d_{ \pm \ell, n_{\perp}} \sqrt{n_{\perp}+\ell+1} e^{i \alpha^{2} t / \mu}-d_{ \pm(\ell+1), n_{\perp}-1}^{*} d_{ \pm \ell, n_{\perp}} \sqrt{n_{\perp}} e^{-i \alpha^{2} t / \mu}\right] \\
& +\frac{1}{\alpha} \sum_{\ell=1}^{\infty} \sum_{n_{\perp}=0}^{\infty}\left[d_{\mp(\ell-1), n_{\perp}}^{*} d_{\mp \ell, n_{\perp}} \sqrt{n_{\perp}+\ell} e^{-i \alpha^{2} t / \mu}-d_{\mp(\ell-1), n_{\perp}+1}^{*} d_{\mp \ell, n_{\perp}} \sqrt{n_{\perp}+1} e^{i \alpha^{2} t / \mu}\right]
\end{aligned}
$$

where we have used Eq. (38).

To proceed we note that $d_{\ell, n_{\perp}}^{*} d_{\ell^{\prime}, n_{\perp}^{\prime}}=d_{-\ell^{\prime}, n_{\perp}^{\prime}}^{*} d_{-\ell, n_{\perp}}$. Now we have after some work

$$
\begin{aligned}
\sum_{\ell=-\infty}^{\infty} \sum_{n_{\perp}=0}^{\infty} \sum_{\ell^{\prime}=-\infty}^{\infty} \sum_{n_{\perp}^{\prime}=0}^{\infty} \int_{\mathbf{K}^{\prime}} d_{m}^{*} d_{n}\left(J_{m n}^{+} \pm J_{m n}^{-}\right) e^{-i\left(E_{n}^{(1)}-E_{m}^{(1)}\right) t} \\
=\frac{2}{\alpha} \sum_{\ell=0}^{\infty} \sum_{n_{\perp}=0}^{\infty}\left[\left(d_{-\ell, n_{\perp}}^{*} d_{-\ell-1, n_{\perp}} \pm d_{\ell, n_{\perp}}^{*} d_{\ell+1, n_{\perp}}\right) \sqrt{n_{\perp}+\ell+1}\right. \\
\left.\quad-\left(d_{-\ell, n_{\perp}+1}^{*} d_{-\ell-1, n_{\perp}} \pm d_{\ell, n_{\perp}+1}^{*} d_{\ell+1, n_{\perp}}\right) \sqrt{n_{\perp}+1}\right] \cos \left(\alpha^{2} t / \mu\right) .
\end{aligned}
$$

Using $d_{\ell, n_{\perp}}=\int d^{2} \mathbf{P}_{\perp} m_{\ell, n_{\perp}}$ with

$$
\begin{aligned}
m_{\ell, n_{\perp}}= & \frac{\tilde{\mathcal{N}}}{(2 \pi)^{3}} \sqrt{\frac{8 \pi^{3 / 2}}{\sigma^{3}}}\left(\frac{2}{|q| B}\right)^{2} \delta_{n_{z}^{0} n_{z}} e^{-\left(K_{z}-P_{z}^{0}\right)^{2} /\left(2 \sigma^{2}\right)} \\
& \times e^{-\left(\mathbf{P}_{\perp}-\mathbf{P}_{\perp}^{0}\right)^{2} /\left(2 \sigma^{2}\right)} \rho^{\left|\ell^{0}\right|} \tilde{\rho}^{|\ell|} e^{i\left(\ell^{0} \phi-\ell \tilde{\phi}\right)} e^{-\frac{1}{2}\left(\gamma^{2} \rho^{2}+\alpha^{2} \tilde{\rho}^{2}\right)} L_{n_{\perp}^{0}}^{\left|\ell^{0}\right|}\left(\gamma^{2} \rho^{2}\right) L_{n_{\perp}}^{|\ell|}\left(\alpha^{2} \tilde{\rho}^{2}\right) \\
& \tilde{\mathcal{N}}=2(2 \pi)^{2} \alpha^{|\ell|+1} \gamma^{\left|\ell^{0}\right|+1} \sqrt{\frac{n_{\perp}^{0} ! n_{\perp} !}{\left(\left|\ell^{0}\right|+n_{\perp}^{0}\right) !\left(|\ell|+n_{\perp}\right) !}}
\end{aligned}
$$

and a recurrence relation for the Laguerre polynomials we can write

$$
\begin{aligned}
\sum_{\ell=-\infty}^{\infty} \sum_{n_{\perp}=0}^{\infty} \sum_{\ell^{\prime}=-\infty}^{\infty} \sum_{n_{\perp}^{\prime}=0}^{\infty} \int_{\mathbf{K}^{\prime}} d_{m}^{*} d_{n}\left(J_{m n}^{+} \pm J_{m n}^{-}\right) e^{-i\left(E_{n}^{(1)}-E_{m}^{(1)}\right) t} \\
=2 \cos \left(\alpha^{2} t / \mu\right) \sum_{\ell=0}^{\infty} \sum_{n_{\perp}=0}^{\infty}\left(d_{-\ell, n_{\perp}}^{*} \int d^{2} \mathbf{P}_{\perp} m_{-\ell-1, n_{\perp}} \tilde{\rho} e^{i \tilde{\phi}} \pm d_{\ell, n_{\perp}}^{*} \int d^{2} \mathbf{P}_{\perp} m_{\ell, n_{\perp}} \tilde{\rho} e^{-i \tilde{\phi}}\right) \\
=2 \cos \left(\alpha^{2} t / \mu\right) \sum_{\ell=0}^{\infty} \sum_{n_{\perp}=0}^{\infty} d_{\ell, n_{\perp}}^{*} \int d^{2} \mathbf{P}_{\perp} m_{\ell, n_{\perp}} \tilde{\rho}\left(e^{i \tilde{\phi}} \pm e^{-i \tilde{\phi}}\right)
\end{aligned}
$$


With this we can obtain

$$
\begin{aligned}
\sum_{\ell=-\infty}^{\infty} \sum_{n_{\perp}=0}^{\infty} \sum_{\ell^{\prime}=-\infty}^{\infty} \sum_{n_{\perp}^{\prime}=0}^{\infty} \int_{\mathbf{K}^{\prime}} d_{m}^{*} d_{n} \mathcal{C}_{m n} e^{-i\left(E_{n}^{(1)}-E_{m}^{(1)}\right) t} & \\
& =2 \cos \left(\alpha^{2} t / \mu\right) \sum_{\ell=0}^{\infty} \sum_{n_{\perp}=0}^{\infty} d_{\ell, n_{\perp}}^{*} \int d^{2} \mathbf{P}_{\perp} m_{\ell, n_{\perp}} \tilde{\rho} \cos \tilde{\phi}
\end{aligned}
$$

and

$$
\begin{aligned}
\sum_{\ell=-\infty}^{\infty} \sum_{n_{\perp}=0}^{\infty} \sum_{\ell^{\prime}=-\infty}^{\infty} \sum_{n_{\perp}^{\prime}=0}^{\infty} \int_{\mathbf{K}^{\prime}} d_{m}^{*} d_{n} \mathcal{S}_{m n} e^{-i\left(E_{n}^{(1)}-E_{m}^{(1)}\right) t} \\
=2 \cos \left(\alpha^{2} t / \mu\right) \sum_{\ell=0}^{\infty} \sum_{n_{\perp}=0}^{\infty} d_{\ell, n_{\perp}}^{*} \int d^{2} \mathbf{P}_{\perp} m_{\ell, n_{\perp}} \tilde{\rho} \sin \tilde{\phi}
\end{aligned}
$$

Recall we are after

$$
\begin{aligned}
\left\langle\mathbf{P}_{\text {kinetic }}\right\rangle & =\sum_{n_{z}=0}^{\infty} \sum_{n_{\perp}=0}^{\infty} \sum_{\ell=-\infty}^{\infty} \int \frac{d^{3} \mathbf{K}}{(2 \pi)^{3}} d_{n}^{*} d_{n}\left[\mathbf{K}-q B\left(\frac{c}{a}, \frac{b}{a}, 0\right)\right] \\
& -q B \sum_{n_{z}^{\prime}=0}^{\infty} \sum_{n_{\perp}^{\prime}=0}^{\infty} \sum_{\ell^{\prime}=-\infty}^{\infty} \int \frac{d^{3} \mathbf{K}^{\prime}}{(2 \pi)^{3}} d_{m}^{*} d_{n}\left(-\mathcal{S}_{m n}, \mathcal{C}_{m n}, 0\right) e^{-i\left(E_{n}^{(1)}-E_{m}^{(1)}\right) t} .
\end{aligned}
$$

Using what we just learned we have

$$
\begin{aligned}
\left\langle\mathbf{P}_{\text {kinetic }}\right\rangle & =\sum_{n_{z}=0}^{\infty} \sum_{n_{\perp}=0}^{\infty} \sum_{\ell=-\infty}^{\infty} \int \frac{d^{3} \mathbf{K}}{(2 \pi)^{3}} d_{n}^{*} d_{n}\left[\mathbf{K}-q B\left(\frac{c}{a}, \frac{b}{a}, 0\right)\right] \\
- & 2 q B \cos \left(\alpha^{2} t / \mu\right) \sum_{n_{z}=0}^{\infty} \sum_{n_{\perp}=0}^{\infty} \sum_{\ell=0}^{\infty} \int \frac{d^{3} \mathbf{K}}{(2 \pi)^{3}} d_{\ell, n_{\perp}}^{*} \int d^{2} \mathbf{P}_{\perp} m_{\ell, n_{\perp}} \tilde{\rho}(-\sin \tilde{\phi}, \cos \tilde{\phi}, 0) .
\end{aligned}
$$

Focusing on the second term, we need to evaluate

$$
\sum_{n_{z}=0}^{\infty} \sum_{n_{\perp}=0}^{\infty} \sum_{\ell=0}^{\infty} \int \frac{d^{3} \mathbf{K}}{(2 \pi)^{3}} d_{\ell, n_{\perp}}^{*} \int d^{2} \mathbf{P}_{\perp} m_{\ell, n_{\perp}} \tilde{\rho}(-\sin \tilde{\phi}, \cos \tilde{\phi}, 0)
$$

The summation over $n_{z}$ and integration over $K_{z}$ can be done analytically. Next, we change integration variables from $\left(\mathbf{K}_{\perp}, \mathbf{P}_{\perp}\right)$ to $(\rho, \phi, \tilde{\rho}, \tilde{\phi})$ and use the completeness of the Lagueere polynomials to eliminate the summation over $n_{\perp}$. Now, one of the integrals over $\tilde{\rho}$ and the summation over $\ell$ can be done analytically. The remaining five integrals are 
evaluated numerically and found to converge to zero. We now have

$$
\begin{gathered}
\left\langle\mathbf{P}_{\text {kinetic }}\right\rangle=\sum_{n_{z}=0}^{\infty} \sum_{n_{\perp}=0}^{\infty} \sum_{\ell=-\infty}^{\infty} \int \frac{d^{3} \mathbf{K}}{(2 \pi)^{3}} d_{n}^{*} d_{n}\left[\mathbf{K}-q B\left(\frac{c}{a}, \frac{b}{a}, 0\right)\right] \\
=\frac{4 \omega_{0}^{2}}{4 \omega_{0}^{2}+\omega_{c}^{2}} \sum_{n_{z}=0}^{\infty} \sum_{n_{\perp}=0}^{\infty} \sum_{\ell=-\infty}^{\infty} \int \frac{d^{3} \mathbf{K}}{(2 \pi)^{3}} d_{n}^{*} d_{n} \mathbf{K}_{\perp} \\
+\hat{z} \sum_{n_{z}=0}^{\infty} \sum_{n_{\perp}=0}^{\infty} \sum_{\ell=-\infty}^{\infty} \int \frac{d^{3} \mathbf{K}}{(2 \pi)^{3}} d_{n}^{*} d_{n} K_{z} .
\end{gathered}
$$

Again, the summation over $n_{z}$ and integration over $K_{z}$ can be done analytically. We change variables, use the completeness of the Lagueere polynomials, and do one of the integrals over $\tilde{\rho}$. Now, we use the completeness of the azimuthal modes and do one of the integrals over $\tilde{\phi}$.

$$
\begin{aligned}
\left\langle\mathbf{P}_{\text {kinetic }}\right\rangle= & \left(\frac{\lambda}{\pi \sigma}\right)^{2} \gamma^{2\left(\left|\ell^{0}\right|+1\right)} \frac{4 \omega_{0}^{2}}{4 \omega_{0}^{2}+\omega_{c}^{2}} \frac{n_{\perp}^{0} !}{\left(\left|\ell^{0}\right|+n_{\perp}^{0}\right) !} \int \rho d \rho \int d \phi \\
& \times \rho^{2\left|\ell^{0}\right|} e^{-\gamma^{2} \rho^{2}}\left(L_{n_{\perp}^{0}\left|\ell^{0}\right|}\left(\gamma^{2} \rho^{2}\right)\right)^{2} \int \tilde{\rho} d \tilde{\rho} \int d \tilde{\phi} \mathbf{K}_{\perp} e^{-\left(\mathbf{P}_{\perp}-\mathbf{P}_{\perp}^{0}\right)^{2} / \sigma^{2}} \\
+ & \hat{z}\left(\frac{\lambda}{\pi \sigma}\right)^{2} \gamma^{2\left(\left|\ell^{0}\right|+1\right)} P_{z}^{0} \frac{n_{\perp}^{0} !}{\left(\left|\ell^{0}\right|+n_{\perp}^{0}\right) !} \int \rho d \rho \int d \phi \\
& \times \rho^{2\left|\ell^{0}\right|} e^{-\gamma^{2} \rho^{2}}\left(L_{n_{\perp}^{0}\left|\ell^{0}\right|}\left(\gamma^{2} \rho^{2}\right)\right)^{2} \int \tilde{\rho} d \tilde{\rho} \int d \tilde{\phi} e^{-\left(\mathbf{P}_{\perp}-\mathbf{P}_{\perp}^{0}\right)^{2} / \sigma^{2}}
\end{aligned}
$$

Using

$$
\begin{aligned}
& P_{x}=-\lambda(\beta y-\tilde{y}), \\
& P_{y}=\lambda(\beta x-\tilde{x}), \\
& K_{x}=-\lambda(y-\tilde{y}), \\
& K_{y}=\lambda(x-\tilde{x}),
\end{aligned}
$$

and the orthogonality of the Laguerre polynomials, the remaining integrals can be done analytically. The final result is

$$
\left\langle\mathbf{P}_{\text {kinetic }}\right\rangle=\left(\frac{4 \omega_{0}^{2}}{4 \omega_{0}^{2}+\omega_{c}^{2}} P_{x}^{0}, \frac{4 \omega_{0}^{2}}{4 \omega_{0}^{2}+\omega_{c}^{2}} P_{y}^{0}, P_{z}^{0}\right)
$$

[1] P. Zeeman, Phil. Mag. 43, 226 (1897).

[2] P. Zeeman, Phil. Mag. 44, 55 (1897). 
[3] P. Zeeman, Nature 44, 347 (1897).

[4] K. Fukushima, D. E. Kharzeev, and H. J. Warringa, Phys.Rev. D78, 074033 (2008), arXiv:0808.3382 [hep-ph].

[5] V. Skokov, A. Y. Illarionov, and V. Toneev, Int.J.Mod.Phys. A24, 5925 (2009), arXiv:0907.1396 [nucl-th],

[6] K. Fukushima, D. E. Kharzeev, and H. J. Warringa, Phys.Rev.Lett. 104, 212001 (2010), arXiv:1002.2495 [hep-ph].

[7] V. Voronyuk, V. Toneev, W. Cassing, E. Bratkovskaya, V. Konchakovski, et al., Phys.Rev. C83, 054911 (2011), arXiv:1103.4239 [nucl-th].

[8] W.-T. Deng and X.-G. Huang, Phys.Rev. C85, 044907 (2012), arXiv:1201.5108 [nucl-th],

[9] K. Tuchin, Adv.High Energy Phys. 2013, 490495 (2013), arXiv:1301.0099 [hep-ph].

[10] R. C. Duncan and C. Thompson, The Astrophysical Journal Letters 392, L9 (1992).

[11] D. T. Son and N. Yamamoto, Phys. Rev. Lett. 109, 181602 (2012).

[12] J.-W. Chen, S. Pu, Q. Wang, and X.-N. Wang, Phys.Rev.Lett. 110, 262301 (2013), arXiv:1210.8312 [hep-th].

[13] M. D'Elia, S. Mukherjee, and F. Sanfilippo, Phys.Rev. D82, 051501 (2010), arXiv:1005.5365 [hep-lat],

[14] M. D'Elia and F. Negro, Phys.Rev. D83, 114028 (2011), arXiv:1103.2080 [hep-lat],

[15] G. Bali, F. Bruckmann, G. Endrodi, Z. Fodor, S. Katz, et al., JHEP 1202, 044 (2012), arXiv:1111.4956 [hep-lat].

[16] G. Bali, F. Bruckmann, G. Endrodi, Z. Fodor, S. Katz, et al., Phys.Rev. D86, 071502 (2012), arXiv:1206.4205 [hep-lat].

[17] G. Bali, F. Bruckmann, G. Endrodi, F. Gruber, and A. Schaefer, JHEP 1304, 130 (2013), arXiv:1303.1328 [hep-lat],

[18] N. Agasian and S. Fedorov, Phys.Lett. B663, 445 (2008), arXiv:0803.3156 [hep-ph],

[19] E. S. Fraga and A. J. Mizher, Phys.Rev. D78, 025016 (2008), arXiv:0804.1452 [hep-ph],

[20] A. J. Mizher, M. Chernodub, and E. S. Fraga, Phys.Rev. D82, 105016 (2010), arXiv:1004.2712 [hep-ph].

[21] K. Fukushima, M. Ruggieri, and R. Gatto, Phys.Rev. D81, 114031 (2010), arXiv:1003.0047 [hep-ph].

[22] R. Gatto and M. Ruggieri, Phys.Rev. D82, 054027 (2010), arXiv:1007.0790 [hep-ph]. 
[23] R. Gatto and M. Ruggieri, Phys.Rev. D83, 034016 (2011), arXiv:1012.1291 [hep-ph],

[24] F. Preis, A. Rebhan, and A. Schmitt, JHEP 1103, 033 (2011), arXiv:1012.4785 [hep-th],

[25] F. Preis, A. Rebhan, and A. Schmitt, J.Phys.G G39, 054006 (2012), arXiv:1109.6904 [hep-th].

[26] J. O. Andersen and R. Khan, Phys.Rev. D85, 065026 (2012), arXiv:1105.1290 [hep-ph],

[27] J. Erdmenger, V. G. Filev, and D. Zoakos, JHEP 1208, 004 (2012), arXiv:1112.4807 [hep-th],

[28] E. Gorbar, V. Miransky, and I. Shovkovy, Prog.Part.Nucl.Phys. 67, 547 (2012), arXiv:1111.3401 [hep-ph].

[29] V. Skokov, Phys.Rev. D85, 034026 (2012), arXiv:1112.5137 [hep-ph],

[30] K. Kashiwa, Phys.Rev. D83, 117901 (2011), arXiv:1104.5167 [hep-ph].

[31] E. S. Fraga and L. F. Palhares, Phys.Rev. D86, 016008 (2012), arXiv:1201.5881 [hep-ph],

[32] E. S. Fraga, J. Noronha, and L. F. Palhares, Phys.Rev. D87, 114014 (2013), arXiv:1207.7094 [hep-ph],

[33] E. S. Fraga, Lect.Notes Phys. 871, 121 (2013), arXiv:1208.0917 [hep-ph].

[34] J. O. Andersen, Phys.Rev. D86, 025020 (2012), arXiv:1202.2051 [hep-ph],

[35] I. A. Shovkovy, Lect.Notes Phys. 871, 13 (2013), arXiv:1207.5081 [hep-ph],

[36] F. Preis, A. Rebhan, and A. Schmitt, Lect.Notes Phys. 871, 51 (2013), arXiv:1208.0536 [hep-ph],

[37] G. N. Ferrari, A. F. Garcia, and M. B. Pinto, Phys.Rev. D86, 096005 (2012), arXiv:1207.3714 [hep-ph].

[38] S. Fayazbakhsh, S. Sadeghian, and N. Sadooghi, Phys.Rev. D86, 085042 (2012), arXiv:1206.6051 [hep-ph].

[39] K. Fukushima and J. M. Pawlowski, Phys.Rev. D86, 076013 (2012), arXiv:1203.4330 [hep-ph],

[40] M. de Paoli and D. Menezes, (2012), arXiv:1203.3175 [nucl-th].

[41] J. Alexandre, Phys.Rev. D63, 073010 (2001), arXiv:hep-th/0009204 [hep-th].

[42] W. E. Lamb and R. C. Retherford, Phys. Rev. 79, 549 (1950).

[43] W. E. Lamb and R. C. Retherford, Phys. Rev. 81, 222 (1951).

[44] W. E. Lamb, Phys. Rev. 85, 259 (1952).

[45] B. P. Carter, Lawrence Radiation Laboratory Report No. UCID-15180 (1967).

[46] L. P. Gor'kov and I. E. Dzyaloshinskii, Soviet Physics JETP 63, 449 (1968).

[47] H. Grotch and R. A. Hegstrom, Phys. Rev. A 4, 59 (1971).

[48] J. Avron, I. Herbst, and B. Simon, Annals of Physics 114, 431 (1978). 
[49] G. Wunner and H. Herold, Astrophysics and Space Science 63, 503 (1979).

[50] V. Pavlov-Verevkin and B. Zhilinskii, Physics Letters A 78, 244 (1980).

[51] J. Avron, I. Herbst, and B. Simon, Communications in Mathematical Physics 79, 529 (1981).

[52] J. E. Avron, I. W. Herbst, and B. Simon, Phys. Rev. A 20, 2287 (1979).

[53] H. Herold, H. Ruder, and G. Wunner, Journal of Physics B: Atomic and Molecular Physics 14, $751(1981)$.

[54] B. R. Johnson, J. O. Hirschfelder, and K.-H. Yang, Rev. Mod. Phys. 55, 109 (1983).

[55] P. Droz-Vincent, Phys. Rev. A 52, 1837 (1995).

[56] H. Georgi, Phys.Lett. B240, 447 (1990),

[57] E. Eichten, K. Gottfried, T. Kinoshita, K. D. Lane, and T.-M. Yan, Phys. Rev. D21, 203 $(1980)$.

[58] W. Lucha, F. F. Schoberl, and D. Gromes, Phys. Rept. 200, 127 (1991)

[59] N. Brambilla, A. Pineda, J. Soto, and A. Vairo, Rev. Mod. Phys. 77, 1423 (2005), arXiv:hepph/0410047.

[60] K. Marasinghe and K. Tuchin, Phys.Rev. C84, 044908 (2011), arXiv:1103.1329 [hep-ph].

[61] K. Tuchin, Phys.Lett. B705, 482 (2011), arXiv:1105.5360 [nucl-th],

[62] D.-L. Yang and B. Muller, J.Phys. G39, 015007 (2012), arXiv:1108.2525 [hep-ph],

[63] Y. Simonov, B. Kerbikov, and M. Andreichikov, (2012), arXiv:1210.0227 [hep-ph].

[64] Y. A. Simonov, (2013), arXiv:1304.0365 [hep-ph],

[65] M. Andreichikov, B. Kerbikov, V. Orlovsky, and Y. A. Simonov, (2013), arXiv:1304.2533 [hep-ph],

[66] C. Machado, F. Navarra, E. de Oliveira, J. Noronha, and M. Strickland, Phys.Rev. D88, 034009 (2013), arXiv:1305.3308 [hep-ph],

[67] J. Pirenne, Arch. Sci. Phys. Nat. 28, 233 (1946).

[68] J. Pirenne, Arch. Sci. Phys. Nat. 29, 121 (1947).

[69] J. Pirenne, Arch. Sci. Phys. Nat. 29, 207 (1947).

[70] J. Pirenne, Arch. Sci. Phys. Nat. 29, 265 (1947).

[71] V. Berestetskii and L. Landau, Zh. Eksp. Teor. Fiz. 19, 673 (1949).

[72] V. Berestetskii and L. Landau, Zh. Eksp. Teor. Fiz. 19, 1130 (1949).

[73] R. A. Ferrell, Phys. Rev. 84, 858 (1951).

[74] M. Deutsch and E. Dulit, Phys. Rev. 84, 601 (1951). 
[75] M. Deutsch and S. C. Brown, Phys. Rev. 85, 1047 (1952).

[76] R. Karplus and A. Klein, Phys. Rev. 87, 848 (1952).

[77] O. Halpern, Phys. Rev. 94, 904 (1954).

[78] V. Hughes, S. Marder, and C. Wu, Phys.Rev. 106, 934 (1957).

[79] M. L. Lewis and V. W. Hughes, Phys. Rev. A 8, 625 (1973),

[80] S. Berko and H. N. Pendleton, Annual Review of Nuclear and Particle Science 30, 543 (1980).

[81] S. G. Karshenboim, Int.J.Mod.Phys. A19, 3879 (2004), arXiv:hep-ph/0310099 [hep-ph].

[82] T. Kawanai and S. Sasaki, Phys.Rev. D85, 091503 (2012), arXiv:1110.0888 [hep-lat],

[83] S. Chatrchyan et al., JHEP 1205, 063 (2012), arXiv:1201.5069 [nucl-ex],

[84] F. Gurin, The European Physical Journal C 49, 143 (2007).

[85] M. Strickland, Phys.Rev.Lett. 107, 132301 (2011), arXiv:1106.2571 [hep-ph].

[86] M. Strickland and D. Bazow, Nucl.Phys. A879, 25 (2012), arXiv:1112.2761 [nucl-th],

[87] K. L. Haglin, (2002), arXiv:nucl-th/0205049 [nucl-th].

[88] T. Barnes, S. Godfrey, and E. Swanson, Phys.Rev. D72, 054026 (2005), arXiv:hep-ph/0505002 [hep-ph].

[89] S. F. Radford and W. W. Repko, Phys.Rev. D75, 074031 (2007), arXiv:hep-ph/0701117 [hepph].

[90] W. W. Repko, M. D. Santia, and S. F. Radford, (2012), arXiv:1211.6373 [hep-ph].

[91] A. Laschka, N. Kaiser, and W. Weise, Phys.Lett. B715, 190 (2012), arXiv:1205.3390 [hep-ph].

[92] J. Beringer et al. (Particle Data Group), Phys.Rev. D86, 010001 (2012).

[93] I. Sudiarta and D. Geldart, Journal of Physics A40, 1885 (2007).

[94] M. Strickland and D. Yager-Elorriaga, J.Comput.Phys. 229, 6015 (2010), arXiv:0904.0939 [quant-ph].

[95] M. Margotta, K. McCarty, C. McGahan, M. Strickland, and D. Yager-Elorriaga, Phys.Rev. D83, 105019 (2011), arXiv:1101.4651 [hep-ph]. 